\title{
Systematic Review \\ Predictive Biomarkers for Outcomes of Immune Checkpoint Inhibitors (ICIs) in Melanoma: A Systematic Review
}

\author{
Joosje C. Baltussen ${ }^{1}\left(\mathbb{D}\right.$, Marij J. P. Welters ${ }^{1,2}$, Elizabeth M. E. Verdegaal ${ }^{1,2}{ }^{\circledR}$, Ellen Kapiteijn ${ }^{1}{ }^{\circledR}$, Anne M. \\ R. Schrader ${ }^{3}$, Marije Slingerland ${ }^{1}$, Gerrit-Jan Liefers ${ }^{4}$, Sjoerd H. van der Burg ${ }^{1,2}{ }^{\mathbb{D}}$, Johanneke E. A. Portielje ${ }^{1}(\mathbb{D})$ \\ and Nienke A. de Glas ${ }^{1, *(\mathbb{D}}$
}

1 Department of Medical Oncology, Leiden University Medical Center, Albinusdreef 2, 2333 ZA Leiden, The Netherlands; J.C.Baltussen@lumc.nl (J.C.B.);

M.J.P.Schoenmaekers-Welters@lumc.nl (M.J.P.W.); E.M.E.Verdegaal@lumc.nl (E.M.E.V.); H.W.Kapiteijn@lumc.nl (E.K.); M.Slingerland@lumc.nl (M.S.); S.H.van_der_Burg@lumc.nl (S.H.v.d.B.); J.E.A.Portielje@lumc.nl (J.E.A.P.)

2 Oncode Institute, Leiden University Medical Center, Albinusdreef 2, 2333 ZA Leiden, The Netherlands

3 Department of Pathology, Leiden University Medical Center, Albinusdreef 2, 2333 ZA Leiden, The Netherlands; A.M.R.Schrader@lumc.nl

4 Department of Surgery, Leiden University Medical Center, Albinusdreef 2, 2333 ZA Leiden, The Netherlands; G.J.Liefers@lumc.nl

* Correspondence: N.A.de_Glas@lumc.nl; Tel.: +31-71-526-3464

Citation: Baltussen, J.C.; Welters, M.J.P.; Verdegaal, E.M.E.; Kapiteijn, E.; Schrader, A.M.R.; Slingerland, M.; Liefers, G.-J.; van der Burg, S.H.; Portielje, J.E.A.; de Glas, N.A. Predictive Biomarkers for Outcomes of Immune Checkpoint Inhibitors (ICIs) in Melanoma: A Systematic Review. Cancers 2021, 13, 6366. https://doi.org/10.3390/ cancers13246366

Academic Editor: David Wong

Received: 26 November 2021 Accepted: 16 December 2021 Published: 18 December 2021

Publisher's Note: MDPI stays neutral with regard to jurisdictional claims in published maps and institutional affiliations.

Copyright: (c) 2021 by the authors. Licensee MDPI, Basel, Switzerland. This article is an open access article distributed under the terms and conditions of the Creative Commons Attribution (CC BY) license (https:// creativecommons.org/licenses/by/ $4.0 /)$.
Simple Summary: Immune checkpoint inhibitors (ICIs) have revolutionized treatment of advanced melanoma and survival of melanoma patients has radically improved since. However, as durable responses after ICIs are only observed in $30-50 \%$ of melanoma patients, there is an unmet need to identify predictive biomarkers for response. This systematic review demonstrates the substantial number of publications that have studied a wide variety of possible biomarkers. Covering 177 publications that investigated 128 unique biomarkers, we provide an overview of all studied biomarkers in correlation with response or survival. We highlight blood, tumor and fecal biomarkers that were associated with response to ICIs in multiple studies. Of these, only T-cell inflamed gene expression profiling was predictive for response in a large clinical trial and validated in other studies, thus representing a promising biomarker for clinical practice. Large validation studies are warranted to confirm the predictive utility of other biomarkers, thereby further personalizing immunotherapy treatment.

Abstract: Immune checkpoint inhibitors (ICIs) have strongly improved the survival of melanoma patients. However, as durable response to ICIs are only seen in a minority, there is an unmet need to identify biomarkers that predict response. Therefore, we provide a systematic review that evaluates all biomarkers studied in association with outcomes of melanoma patients receiving ICIs. We searched Pubmed, COCHRANE Library, Embase, Emcare, and Web of Science for relevant articles that were published before June 2020 and studied blood, tumor, or fecal biomarkers that predicted response or survival in melanoma patients treated with ICIs. Of the 2536 identified reports, 177 were included in our review. Risk of bias was high in $40 \%$, moderate in $50 \%$ and low in $10 \%$ of all studies. Biomarkers that correlated with response were myeloid-derived suppressor cells (MDSCs), circulating tumor cells (CTCs), CD8+ memory T-cells, T-cell receptor (TCR) diversity, tumor-infiltrating lymphocytes (TILs), gene expression profiling (GEP), and a favorable gut microbiome. This review shows that biomarkers for ICIs in melanoma patients are widely studied, but heterogeneity between studies is high, average sample sizes are low, and validation is often lacking. Future studies are needed to further investigate the predictive utility of some promising candidate biomarkers.

Keywords: melanoma; immune checkpoint inhibitor; prediction; response; biomarkers; systematic review 


\section{Introduction}

Metastatic melanoma is a tumor with poor prognosis and the incidence will continue to rise in the years to come. In the Netherlands, the incidence of melanoma almost tripled in twenty years, from 2525 patients in 2000 to 6787 in 2020 [1]. However, since the introduction of novel therapeutic agents, such as immunotherapy with immune checkpoint inhibitors (ICIs) and targeted therapy, survival of patients with advanced melanoma has radically improved. Immune checkpoints, proteins expressed by T-cells, regulate T-cell functionality and act as gatekeepers of immune responses to prevent autoimmunity. Immune checkpoint inhibitors aim to unleash the compromised T-cells, thereby inducing an anti-tumor response [2].

The first ICI approved by the US Food and Drug Administration (FDA) in 2011 was ipilimumab, a cytotoxic-T-lymphocyte-associated-protein-4 (CTLA-4) targeting antibody. Anti-CTLA-4 antibodies block T-cell inhibition, thereby promoting T-cell activation and the anti-tumor response. Survival increased from 6.4 months to 10 months for melanoma patients treated with ipilimumab compared to a vaccine control or dacarbazine. Moreover, survival curves seemed to reach a plateau between two and three years, demonstrating that a proportion of patients experienced a sustained long-term survival. Yet, response rates were lower than $20 \%$ and immune-related adverse events grade 3-4 were seen in approximately $15-45 \%$ of all patients [3]. More promising results were seen in trials comparing ipilimumab to nivolumab or pembrolizumab, the latter which are anti-programmed death-1 (PD-1) antibodies. Anti-PD-1 antibodies aim to prevent inhibitory signaling in activated effector T-cells by blocking their binding to PD-L1/2, resulting in functional Tcells that are possibly able to kill tumor cells. Objective responses ranged from $20-30 \%$ for monotherapy to $40-50 \%$ for combination therapy of anti-CTLA-4 and anti-PD-1. Furthermore, grade 3-4 toxicity dropped to $11-16 \%$ for anti-PD-1 monotherapy. In combination therapy, however, grade 3-4 toxicity rates remain at $40 \%$ due to anti-CTLA-4 therapy [4-6].

Although ICIs, specifically anti-PD-1 therapy, show better response rates than previous therapies, durable response is still seen in a subset of patients and risk of severe toxicity, such as diarrhea, hepatotoxicity and skin rash, remains high, especially in combination treatment. Biomarkers are therefore needed to predict response to therapy, thereby maximizing the therapeutic benefit for potential responders and saving toxicity and high costs for patients that are unlikely to benefit from ICIs. Biomarkers associated with response to ICI therapy in melanoma patients range from lactate dehydrogenase (LDH) in peripheral blood to tumor mutation burden (TMB), PD-L1 immunohistochemical positivity, and tumor-infiltrating lymphocytes (TILs) in tumor tissue [7-10]. Apart from LDH, these markers have not yet been integrated into standard clinical care. Therefore, this systematic review aimed to provide a full overview of the evidence for blood, tumor and fecal biomarkers that correlate with response, progression-free survival, and overall survival for ICIs in metastatic melanoma patients.

\section{Materials and Methods}

\subsection{Search Strategy}

On the 18 June 2020, we searched in the following electronic bibliographic databases for our systematic review: PubMed, COCHRANE Library, Embase, Emcare, and Web of Science. The search strategy used a combination of the following terms: "melanoma", "immune checkpoint inhibitors" OR "immunotherapy", "response" OR "outcome", "predictor" and "biomarkers" (complete search strategy in Supplementary Material). The search was performed together with a research librarian. Duplicates were removed in EndNote. Two investigators (J.B and N.G) independently screened titles and abstracts to determine eligibility. Together with J.P., the same investigators independently assessed the full-text articles of potentially relevant studies to verify if eligibility criteria were met. All full texts were appraised by two authors. Any disagreement was resolved by a third author. 


\subsection{Selection Criteria}

We aimed to identify studies in advanced melanoma patients receiving ICIs that investigated the association between blood, tumor, and fecal biomarkers with outcomes. Included studies had to study either anti-CLTA-4 treatment (ipilimumab), anti-PD-1 (nivolumab, pembrolizumab,) or combination therapy. Included study designs were randomized clinical trials, cohort studies (both retrospective and prospective), and case-control studies. Baseline biomarkers as well as biomarkers during treatment were considered eligible. Outcomes of interest were response (both objective response rate (ORR) and clinical benefit), overall survival (OS), melanoma-specific survival (MSS), and progression-free survival (PFS). Systematic reviews, editorials, and case reports were excluded, as well as articles that were not written in English, animal studies, and reports studying the ICI tremelimumab $(N=1)$, as this therapy proved not to be effective [11]. We excluded publications that analyzed biomarkers in adjuvant chemotherapy, interleukins, vaccination treatment, or targeted therapy. Reports studying adverse events or pseudoprogression as a single outcome and reports studying imaging biomarkers were not eligible.

\subsection{Data Extraction and Quality Assessment}

This systematic review was reported following the Preferred Reporting Items for Systematic Reviews and Meta-analyses (PRISMA) reporting guidelines [12]. Study characteristics, including first author, journal, publication date, study design and phase, type and timing of biomarker, type of therapy, baseline patient characteristics such as type of melanoma, age, country, and follow-up, and outcomes were extracted. We reported both positive and negative study results. Due to heterogeneity of the included studies, a meta-analysis was not considered feasible.

We estimated risk of bias by using the QUIPS tool, a quality tool for prognostic factor studies that examines risk of bias by the following six domains: study participation, study attrition, prognostic factor measurement, outcome measurement, adjustment for other prognostic factors, and statistical analysis [13]. We classified risk of bias for adjustment as low if researchers adjusted outcomes for at least tumor load or LDH and metastasis for response and age or WHO status for survival, as these factors have shown to be important confounders. Selected thresholds and the rationale for these thresholds had to be mentioned to score a low risk of bias for prognostic factor measurement. Statistical analyses were assessed as high risk of bias if only $p$-values were mentioned, and a 95\% confidence interval (CI) was not calculated. In case of disagreement between two authors, consensus was reached after discussion. We considered risk of bias high if three or more categories were reported as high risk [14]. When studies scored low in all categories or at least in the categories of attrition, outcome measurement and statistical reporting, the risk was defined as low. All other studies were scored as moderate risk of bias.

\subsection{Data Presentation}

Study characteristics and outcomes are presented in tables and separated into antiCTLA-4, anti-PD-1, combination therapy and mixed therapy cohorts. Mixed cohorts included both patients receiving anti-CTLA-4, anti-PD-1 and combination therapy. Biomarkers that were investigated in more than one study are shown in graphs. The median number of included patients per biomarker with interquartile range (IQR) was calculated. Graphs were created with GraphPad PRISM 9.0.1 and BioRender.com (accessed on 24 November 2021) was used to create Figure 4.

\section{Results}

The initial literature search yielded 3940 publications, of which 1404 were duplicates. Of the unique 2536 publications, 1996 records were identified in PubMed, 389 in Web of Science, 21 in COCHRANE library and 130 in Embase. After title and abstract screening based on inclusion criteria as defined in the Methods section, 267 studies were eligible for our review. We extracted the full text of these publications and after screening thereof, we 
excluded 106 studies and 161 studies remained suitable for our review. Cross referencing identified another 16 relevant publications, which resulted in 175 original publications that we included in our review (Figure 1, Supplementary Material for complete reference list).

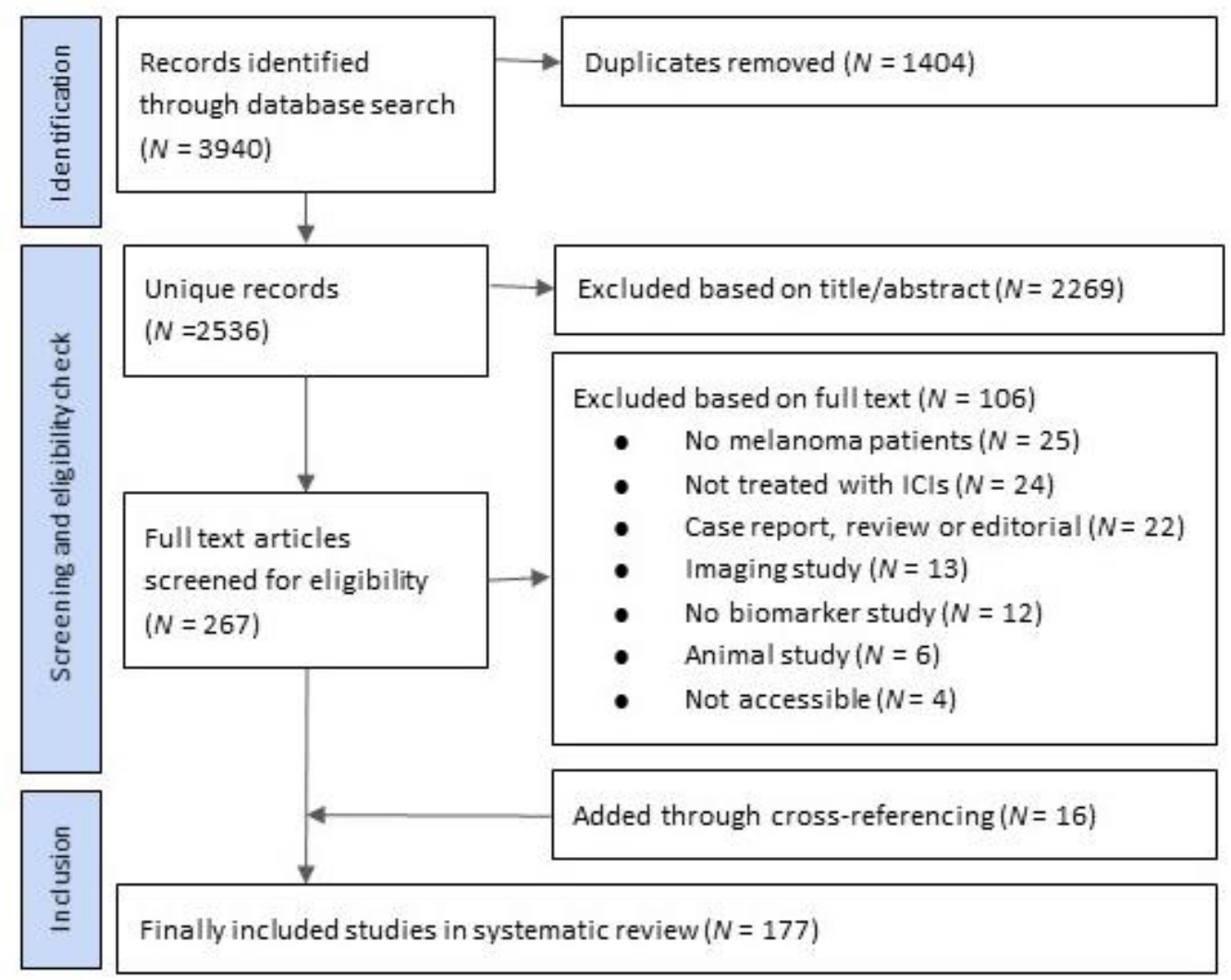

Figure 1. Study selection based on PRISMA methods.

A description of the 177 identified reports is shown in supplemental Tables S1 and S2. Of all publications, $77 \%(N=136)$ reported response as outcome, $66 \%(N=117)$ studied OS and in $43 \%(N=76)$ of all publications PFS was studied. Adjustment for confounders was performed in 69 articles. We found 24 reports that analyzed parameters in a development cohort as well as a validation cohort and 153 reports that only investigated biomarkers in a developmental study design. In most studies, $55-70 \%$ of the included patients were men. Information about race was only described in five studies.

In total, we identified 128 unique biomarkers. Most studies investigated biomarkers for anti-PD-1 therapy $(N=81)$ and anti-CTLA-4 therapy $(N=73)$, whereas 12 publications studied combination therapy (Tables 1-4). Moreover, 24 publications were based on mixed cohorts with both anti-CTLA-4, anti-PD-1, and combination therapy (Table 4). Biomarkers were most frequently investigated before the start of treatment $(N=103)$, 71 reports analyzed them before and during treatment and three reports only measured markers during treatment. We assessed the quality and found that $40 \%$ of all publications $(N=70)$ were estimated high risk of bias, $50 \%(N=89)$ were moderate risk of bias and $10 \%$ $(N=18)$ were estimated low risk of bias. Publications with low risk of bias mainly studied blood biomarkers. 
Table 1. Summary of most important biomarkers that were studied for anti-CTLA-4 therapy and assessed risk of bias per article.

\begin{tabular}{|c|c|c|c|c|c|c|c|}
\hline Biomarker & $\begin{array}{c}N \text { of } \\
\text { Studies }\end{array}$ & $\begin{array}{c}N \text { of } \\
\text { Patients }\end{array}$ & $\begin{array}{c}\text { Median } \\
\text { Patients per } \\
\text { Article (IQR) }\end{array}$ & Response & PFS & OS & $\begin{array}{c}\text { Quality } \\
\text { Assessment }\end{array}$ \\
\hline $\mathrm{LDH}$ & 20 & 2539 & $86(53-86)$ & $\begin{array}{l}\text { LDH was associated } \\
\text { with response in } 4 / 10 \\
\text { studies, not associated } \\
\text { in } 6 / 10 \text { studies. }\end{array}$ & $\begin{array}{c}\text { LDH was } \\
\text { associated with } \\
\text { PFS in } 3 / 3 \text { studies. }\end{array}$ & $\begin{array}{l}\text { LDH was associated } \\
\text { with OS in } 12 / 16 \\
\text { studies, not associated } \\
\text { in } 4 / 16 \text { studies. }\end{array}$ & $\begin{array}{l}2 / 20 \text { high risk, } \\
14 / 20 \text {, moderate, } \\
4 / 20 \text { low risk of } \\
\text { bias }\end{array}$ \\
\hline NLR & 11 & 1632 & $78(43-184)$ & $\begin{array}{l}\text { NLR was associated } \\
\text { with response in } 3 / 5 \\
\text { studies, not associated } \\
\text { in } 2 / 5 \text { studies. }\end{array}$ & $\begin{array}{c}\text { NLR was } \\
\text { associated with } \\
\text { PFS in } 4 / 5 \text { studies, } \\
\text { not associated in } \\
1 / 5 \text { studies. }\end{array}$ & $\begin{array}{c}\text { NLR was associated } \\
\text { with OS in } 7 / 10 \text { studies, } \\
\text { not associated in } 3 / 10 \\
\text { studies }\end{array}$ & $\begin{array}{c}\text { 1/11 high risk, } \\
6 / 11 \text { moderate risk, } \\
4 / 11 \text { low risk of } \\
\text { bias }\end{array}$ \\
\hline TMB & 7 & 724 & $64(56-174)$ & $\begin{array}{c}\text { TMB was associated } \\
\text { with response in } 3 / 6 \\
\text { studies, not associated } \\
\text { in } 3 / 6 \text { studies. }\end{array}$ & & $\begin{array}{c}\text { TMB was associated } \\
\text { with OS in } 2 / 4 \text { studies, } \\
\text { not associated in } 2 / 4 \\
\text { studies. }\end{array}$ & $\begin{array}{c}\text { 3/7 high risk, } \\
4 / 7 \text { moderate, } \\
0 / 7 \text { low risk of bias }\end{array}$ \\
\hline $\begin{array}{l}\text { Neoantigen } \\
\text { load (NAL) }\end{array}$ & 5 & 385 & $64(54-107)$ & $\begin{array}{l}\text { NAL was associated } \\
\text { with response in } 2 / 3 \\
\text { studies, not associated } \\
\text { in } 1 / 3 \text { studies. }\end{array}$ & & $\begin{array}{l}\text { NAL was associated } \\
\text { with OS in } 2 / 2 \text { studies. }\end{array}$ & $\begin{array}{c}2 / 5 \text { high risk } \\
3 / 5 \text { moderate, } \\
0 / 5 \text { low risk of bias }\end{array}$ \\
\hline $\begin{array}{l}\text { PD-L1 } \\
\text { expression } \\
\text { on tumor } \\
\text { cells }\end{array}$ & 5 & 637 & $111(48-214)$ & $\begin{array}{l}\text { PD-L1 was not } \\
\text { associated with } \\
\text { response in } 5 / 5 \text { studies. }\end{array}$ & $\begin{array}{l}\text { PD-L1 was not } \\
\text { associated with } \\
\text { PFS in } 1 / 1 \text { studies. }\end{array}$ & $\begin{array}{c}\text { PDL-1 was not } \\
\text { associated with OS in } \\
3 / 3 \text { studies. }\end{array}$ & $\begin{array}{c}2 / 5 \text { high risk, } \\
3 / 5 \text { moderate, } 0 / 5 \\
\text { low risk of bias }\end{array}$ \\
\hline MDSCs & 4 & 726 & $48(22-475)$ & $\begin{array}{l}\text { MDSCs were associated } \\
\text { with response in } 3 / 3 \\
\text { studies. }\end{array}$ & & $\begin{array}{l}\text { MDSCs were associated } \\
\text { with OS in } 2 / 2 \text { studies. }\end{array}$ & $\begin{array}{c}1 / 4 \text { high risk, } \\
3 / 4 \text { moderate risk, } \\
0 / 3 \text { low risk of bias }\end{array}$ \\
\hline $\begin{array}{l}\text { T-cell } \\
\text { inflamed } \\
\text { GEP }\end{array}$ & 4 & 304 & $58(33-192)$ & $\begin{array}{l}\text { GEP was associated } \\
\text { with response in } 4 / 4 \\
\text { studies. }\end{array}$ & & & $\begin{array}{l}2 / 4 \text { high risk, } 2 / 4 \\
\text { moderate, } 0 / 4 \text { low } \\
\text { risk of bias }\end{array}$ \\
\hline $\begin{array}{l}\text { Tregs in } \\
\text { tumor tissue }\end{array}$ & 4 & 169 & $38(31-58)$ & $\begin{array}{l}\text { Tregs were associated } \\
\text { with response in } 2 / 4 \\
\text { studies, not associated } \\
\text { in } 2 / 4 \text { studies. }\end{array}$ & & $\begin{array}{c}\text { Tregs were associated } \\
\text { with OS in } 2 / 2 \text { studies. }\end{array}$ & $\begin{array}{c}\text { 0/4 high risk, } \\
4 / 4 \text { moderate, } \\
0 / 4 \text { low risk of bias }\end{array}$ \\
\hline $\begin{array}{l}\text { monocytic } \\
\text { MDSCs }\end{array}$ & 4 & 168 & $39(32-55)$ & $\begin{array}{l}\text { moMDSCs were } \\
\text { associated with } \\
\text { response in } 2 / 2 \text { studies. }\end{array}$ & $\begin{array}{l}\text { moMDSCs were } \\
\text { associated with } \\
\text { PFS in } 2 / 2 \text { studies }\end{array}$ & $\begin{array}{c}\text { moMDSCs were } \\
\text { associated with OS in } \\
1 / 1 \text { studies. }\end{array}$ & $\begin{array}{c}3 / 4 \text { high risk } \\
1 / 4 \text { moderate risk, } \\
0 / 4 \text { low risk of bias }\end{array}$ \\
\hline $\begin{array}{l}\text { Tregs in } \\
\text { blood }\end{array}$ & 3 & 741 & $95(31-615)$ & $\begin{array}{l}\text { Tregs were associated } \\
\text { with response in } 1 / 1 \\
\text { studies. }\end{array}$ & $\begin{array}{c}\text { Tregs were } \\
\text { associated with } \\
\text { RFS in } 1 / 1 \text { studies }\end{array}$ & $\begin{array}{l}\text { Tregs were associated } \\
\text { with OS in } 2 / 2 \text { studies. }\end{array}$ & $\begin{array}{c}1 / 3 \text { high risk, } \\
2 / 3 \text { moderate risk, } \\
0 / 3 \text { as low risk of } \\
\text { bias }\end{array}$ \\
\hline $\begin{array}{l}\text { CD8 } \\
\text { memory } \\
\text { T-cells in } \\
\text { blood }\end{array}$ & 3 & 90 & $30(17-43)$ & $\begin{array}{l}\text { CD8 memory T-cells } \\
\text { were associated with } \\
\text { response in } 2 / 2 \text { studies. }\end{array}$ & & $\begin{array}{l}\text { CD8 memory T-cells } \\
\text { were associated with OS } \\
\text { in } 3 / 3 \text { studies. }\end{array}$ & $\begin{array}{c}2 / 3 \text { high risk, } 1 / 3 \\
\text { moderate risk } 0 / 1 \\
\text { low risk of bias }\end{array}$ \\
\hline TILs & 3 & 90 & $17(9-64)$ & $\begin{array}{l}\text { TILs were associated } \\
\text { with response in } 3 / 3 \\
\text { studies. }\end{array}$ & $\begin{array}{c}\text { TILs were not } \\
\text { associated with } \\
\text { PFS in } 1 / 1 \text { studies. }\end{array}$ & $\begin{array}{c}\text { TILs were not } \\
\text { associated with OS in } \\
1 / 1 \text { studies. }\end{array}$ & $\begin{array}{c}1 / 3 \text { high risk, } \\
2 / 3 \text { moderate, } 0 / 3 \\
\text { low risk of bias }\end{array}$ \\
\hline $\begin{array}{l}\text { TCR } \\
\text { diversity in } \\
\text { blood }\end{array}$ & 2 & 54 & 27 (N/A) & $\begin{array}{l}\text { TCR diversity was } \\
\text { associated with } \\
\text { response in } 2 / 2 \text { studies. }\end{array}$ & & $\begin{array}{c}\text { TCR diversity was not } \\
\text { associated with OS in } \\
1 / 1 \text { studies. }\end{array}$ & $\begin{array}{c}1 / 2 \text { high risk, } \\
1 / 2 \text { moderate risk, } \\
0 / 2 \text { low risk of bias }\end{array}$ \\
\hline $\begin{array}{l}\text { NK cells in } \\
\text { blood }\end{array}$ & 2 & 63 & 32 (N/A) & $\begin{array}{c}\text { associated with } \\
\text { response in } 1 / 2 \text { studies, } \\
\text { not associated in } 1 / 2 \\
\text { studies. }\end{array}$ & & & $\begin{array}{c}1 / 2 \text { high risk, } \\
1 / 2 \text { moderate, risk } \\
0 / 2 \text { low risk of bias }\end{array}$ \\
\hline
\end{tabular}

Abbreviations: GEP: gene expression profiling, IQR: interquartile range, LDH: lactate dehydrogenase, MDSCs: myeloid-derived suppressor cells, NK: natural killer, NLR: neutrophil-to-lymphocyte ratio, TCR: T-cell receptor, TILs: tumor-infiltrating lymphocytes, TMB: tumor mutation burden, Tregs: regulatory T cells.

Table 2. Summary of most important biomarkers that were studied for anti-PD-1 therapy and assessed risk of bias per article.

\begin{tabular}{|c|c|c|c|c|c|c|c|}
\hline Biomarker & $\begin{array}{c}N \text { of } \\
\text { Studies }\end{array}$ & $\begin{array}{c}\mathrm{N} \text { of } \\
\text { Patients }\end{array}$ & $\begin{array}{c}\text { Median } \\
\text { Patients per } \\
\text { Study (IQR) }\end{array}$ & Response & PFS & OS & $\begin{array}{c}\text { Quality } \\
\text { Assessment }\end{array}$ \\
\hline $\mathrm{LDH}$ & 20 & 2274 & 78 (39-152) & $\begin{array}{l}\text { LDH was associated } \\
\text { with response in } 4 / 10 \\
\text { studies, not associated } \\
\text { in } 6 / 10 \text { studies. }\end{array}$ & $\begin{array}{c}\text { LDH was } \\
\text { associated with } \\
\text { PFS in } 10 / 11 \\
\text { studies, not } \\
\text { associated in } 1 / 11 \\
\text { studies. }\end{array}$ & $\begin{array}{c}\text { LDH was associated } \\
\text { with OS in } 13 / 13 \\
\text { studies. }\end{array}$ & $\begin{array}{c}5 / 20 \text { high, } \\
12 / 20 \text { moderate, } \\
3 / 20 \text { low risk of } \\
\text { bias }\end{array}$ \\
\hline $\begin{array}{l}\text { PD-L1 } \\
\text { expression } \\
\text { on tumor } \\
\text { cells }\end{array}$ & 12 & 1481 & $52(30-68)$ & $\begin{array}{l}\text { PD-L1 was associated } \\
\text { with response in } 7 / 12 \\
\text { studies, not associated } \\
\text { in } 5 / 12 \text { studies. }\end{array}$ & $\begin{array}{l}\text { PD-L1 was } \\
\text { associated with } \\
\text { PFS in } 2 / 5 \text { studies, } \\
\text { not associated in } \\
3 / 5 \text { studies. }\end{array}$ & $\begin{array}{c}\text { PD-L1 was associated } \\
\text { with OS in } 3 / 4 \text { studies, } \\
\text { not associated in } 1 / 4 \\
\text { studies. }\end{array}$ & $\begin{array}{c}8 / 12 \text { high, } \\
4 / 12 \text { moderate, } \\
0 / 12 \text { low risk of } \\
\text { bias }\end{array}$ \\
\hline
\end{tabular}


Table 2. Cont.

\begin{tabular}{|c|c|c|c|c|c|c|c|}
\hline Biomarker & $\begin{array}{l}N \text { of } \\
\text { Studies }\end{array}$ & $\begin{array}{c}\mathrm{N} \text { of } \\
\text { Patients }\end{array}$ & $\begin{array}{c}\text { Median } \\
\text { Patients per } \\
\text { Study (IQR) }\end{array}$ & Response & PFS & OS & $\begin{array}{c}\text { Quality } \\
\text { Assessment }\end{array}$ \\
\hline $\begin{array}{l}\text { T-cell } \\
\text { inflamed } \\
\text { GEP }\end{array}$ & 9 & 1237 & $58(33-192)$ & $\begin{array}{c}\text { GEP was associated } \\
\text { with response in } 7 / 9 \\
\text { studies, not } \\
\text { associated in } 2 / 9 \\
\text { studies. }\end{array}$ & $\begin{array}{c}\text { GEP was } \\
\text { associated with } \\
\text { PFS in } 1 / 2 \\
\text { studies, not } \\
\text { associated in } 1 / 2 \\
\text { studies }\end{array}$ & $\begin{array}{l}\text { GEP was not } \\
\text { associated with OS in } \\
2 / 2 \text { studies. }\end{array}$ & $\begin{array}{c}\text { 4/9 high, } \\
5 / 9 \text { moderate, } \\
0 / 9 \text { low risk of } \\
\text { bias }\end{array}$ \\
\hline NLR & 8 & 732 & 77 (41-138) & $\begin{array}{l}\text { NLR was associated } \\
\text { with response } 1 / 3 \\
\text { studies, not } \\
\text { associated in } 2 / 3 \\
\text { studies. }\end{array}$ & $\begin{array}{l}\text { NLR was } \\
\text { associated with } \\
\text { PFS in } 5 / 5 \\
\text { studies. }\end{array}$ & $\begin{array}{l}\text { NLR was associated } \\
\text { with OS in } 6 / 6 \\
\text { studies. }\end{array}$ & $\begin{array}{c}1 / 8 \text { high, } \\
6 / 8 \text { moderate, } \\
1 / 8 \text { low risk of } \\
\text { bias }\end{array}$ \\
\hline TMB & 8 & 68 & $52(41-67)$ & $\begin{array}{l}\text { TMB was associated } \\
\text { with response in } 3 / 6 \\
\text { studies, not } \\
\text { associated in } 3 / 6 \\
\text { studies. } \\
\text { NK cells were }\end{array}$ & $\begin{array}{l}\text { TMB was } \\
\text { associated with } \\
\text { PFS in } 2 / 2 \\
\text { studies. }\end{array}$ & $\begin{array}{l}\text { TMB was associated } \\
\text { with OS in } 3 / 4 \\
\text { studies, not } \\
\text { associated in } 1 / 4 \\
\text { studies. }\end{array}$ & $\begin{array}{c}\text { 4/8 high, } \\
4 / 8 \text { moderate, } \\
0 / 8 \text { low risk of } \\
\text { bias }\end{array}$ \\
\hline $\begin{array}{l}\text { NK cells in } \\
\text { blood }\end{array}$ & 5 & 128 & $20(13-41)$ & $\begin{array}{l}\text { associated with } \\
\text { response in } 3 / 4 \\
\text { studies, not } \\
\text { associated in } 1 / 4 \\
\text { studies. }\end{array}$ & & $\begin{array}{l}\text { NK cells were not } \\
\text { associated with OS in } \\
2 / 2 \text { studies. }\end{array}$ & $\begin{array}{c}4 / 5 \text { high, } \\
1 / 5 \text { moderate, } \\
0 / 5 \text { low risk of } \\
\text { bias }\end{array}$ \\
\hline $\begin{array}{c}\text { TCR } \\
\text { diversity } \\
\text { in tumor }\end{array}$ & 4 & 184 & $52(22-57)$ & $\begin{array}{l}\text { TCR diversity was } \\
\text { associated with } \\
\text { response in } 3 / 4 \\
\text { studies, not } \\
\text { associated in } 1 / 4 \\
\text { studies. }\end{array}$ & & & $\begin{array}{c}\text { 2/4 high, } \\
2 / 4 \text { moderate, } \\
0 / 4 \text { low risk of } \\
\text { bias }\end{array}$ \\
\hline $\begin{array}{l}\text { Gut micro- } \\
\text { biomes }\end{array}$ & 2 & 104 & $52(\mathrm{~N} / \mathrm{A})$ & $\begin{array}{l}\text { Gut microbiomes } \\
\text { were associated with } \\
\text { response in } 2 / 2 \\
\text { studies }\end{array}$ & $\begin{array}{l}\text { Gut microbiomes } \\
\text { were associated } \\
\text { with PFS in } 1 / 1 \\
\text { studies }\end{array}$ & & $\begin{array}{c}0 / 2 \text { high, } \\
2 / 2 \text { moderate, } \\
0 / 2 \text { low risk }\end{array}$ \\
\hline $\begin{array}{c}\text { CD8 } \\
\text { memory } \\
\text { T-cells in } \\
\text { blood }\end{array}$ & 2 & 29 & $15(\mathrm{~N} / \mathrm{A})$ & $\begin{array}{l}\text { CD8 memory cells } \\
\text { were associated with } \\
\text { response in } 1 / 2 \\
\text { studies, not } \\
\text { associated in } 1 / 2 \\
\text { studies. }\end{array}$ & & $\begin{array}{c}\text { CD8 memory cells } \\
\text { were not associated } \\
\text { with OS in } 1 / 1 \\
\text { studies. }\end{array}$ & $\begin{array}{c}2 / 2 \text { high, } \\
0 / 2 \text { moderate, } \\
0 / 2 \text { low risk of } \\
\text { bias }\end{array}$ \\
\hline TILs & 2 & 121 & $60(\mathrm{~N} / \mathrm{A})$ & $\begin{array}{l}\text { TILs were associated } \\
\text { with response in } 2 / 2 \\
\text { studies }\end{array}$ & & & $\begin{array}{c}2 / 2 \text { high, } \\
0 / 2 \text { moderate, } \\
0 / 2 \text { low risk of } \\
\text { bias }\end{array}$ \\
\hline ctDNA & 1 & 85 & N/A & & $\begin{array}{l}\text { ctDNA was } \\
\text { associated with } \\
\text { PFS in } 1 / 1 \\
\text { studies. }\end{array}$ & $\begin{array}{c}\text { ctDNA was } \\
\text { associated with OS in } \\
1 / 1 \text { studies. }\end{array}$ & $\begin{array}{c}\text { 0/1 high, } \\
1 / 1 \text { moderate, } \\
0 / 1 \text { low risk of } \\
\text { bias }\end{array}$ \\
\hline MDSCs & 1 & 92 & $\mathrm{~N} / \mathrm{A}$ & $\begin{array}{l}\text { MDSCs were } \\
\text { associated with } \\
\text { response in } 1 / 1 \\
\text { studies. }\end{array}$ & $\begin{array}{l}\text { MDSCs were } \\
\text { associated with } \\
\text { PFS in } 1 / 1 \\
\text { studies. }\end{array}$ & $\begin{array}{l}\text { MDSCs were } \\
\text { associated with OS in } \\
1 / 1 \text { studies. }\end{array}$ & $\begin{array}{c}\text { 0/1 high, } \\
1 / 1 \text { moderate, } \\
0 / 1 \text { low risk of } \\
\text { bias }\end{array}$ \\
\hline $\begin{array}{l}\text { Tregs in } \\
\text { blood }\end{array}$ & 1 & 46 & N/A & $\begin{array}{l}\text { Tregs were not } \\
\text { associated with } \\
\text { response in } 1 / 1 \\
\text { studies. }\end{array}$ & & & $\begin{array}{c}1 / 1 \text { high, } \\
0 / 1 \text { moderate, } \\
0 / 1 \text { low risk of } \\
\text { bias }\end{array}$ \\
\hline $\begin{array}{c}\text { TCR } \\
\text { diversity } \\
\text { in blood }\end{array}$ & 1 & 38 & $\mathrm{~N} / \mathrm{A}$ & $\begin{array}{l}\text { TCR diversity was } \\
\text { associated with } \\
\text { response in } 1 / 1 \\
\text { studies. }\end{array}$ & & & $\begin{array}{c}0 / 1 \text { high, } \\
1 / 1 \text { moderate, } \\
0 / 1 \text { low risk of } \\
\text { bias }\end{array}$ \\
\hline
\end{tabular}

Abbreviations: ctDNA: circulating tumor DNA, GEP: gene expression profiling, IQR: interquartile range, LDH: lactate dehydrogenase, MDSCs: myeloid-derived suppressor cells, NK: natural killer, NLR: neutrophil-to-lymphocyte ratio, TCR: T-cell receptor, TILs: tumorinfiltrating lymphocytes, TMB: tumor mutation burden, Tregs: regulatory T cells. 
Table 3. Summary of most important biomarkers that were studied for combination therapy and assessed risk of bias per article.

\begin{tabular}{|c|c|c|c|c|c|c|c|}
\hline Biomarker & $\begin{array}{c}\mathbf{N} \text { of } \\
\text { Studies }\end{array}$ & $\begin{array}{c}\mathrm{N} \text { of } \\
\text { Patients }\end{array}$ & $\begin{array}{c}\text { Median } \\
\text { Patients per } \\
\text { Study (IQR) }\end{array}$ & Response & PFS & OS & $\begin{array}{c}\text { Quality } \\
\text { Assessment }\end{array}$ \\
\hline $\mathrm{LDH}$ & 2 & 295 & 148 & $\begin{array}{c}\text { LDH was associated } \\
\text { with response in } 1 / 2 \\
\text { studies, not } \\
\text { associated in } 1 / 2 \\
\text { studies. }\end{array}$ & $\begin{array}{l}\text { LDH was } \\
\text { associated with } \\
\text { PFS in } 1 / 1 \\
\text { studies. }\end{array}$ & $\begin{array}{c}\text { LDH was } \\
\text { associated with OS } \\
\text { in } 2 / 2 \text { studies. }\end{array}$ & $\begin{array}{c}0 / 2 \text { high risk, } \\
1 / 2 \text { moderate, } \\
1 / 2 \text { low risk of } \\
\text { bias }\end{array}$ \\
\hline NLR & 1 & 209 & $\mathrm{~N} / \mathrm{A}$ & $\begin{array}{c}\text { NLR was not } \\
\text { associated with } \\
\text { response in } 1 / 1 \\
\text { studies. }\end{array}$ & & $\begin{array}{c}\text { NLR was } \\
\text { associated with OS } \\
\text { in } 1 / 1 \text { studies. }\end{array}$ & $\begin{array}{c}0 / 1 \text { high risk, } \\
1 / 1 \text { moderate } \\
0 / 1 \text { low risk of } \\
\text { bias }\end{array}$ \\
\hline $\begin{array}{c}\text { TCR } \\
\text { diversity }\end{array}$ & 1 & 80 & $\mathrm{~N} / \mathrm{A}$ & & $\begin{array}{c}\text { TCR diversity } \\
\text { was associated } \\
\text { with PFS in } 1 / 1 \\
\text { studies. }\end{array}$ & & $\begin{array}{c}0 / 1 \text { high risk } \\
1 / 1 \text { moderate, } \\
0 / 1 \text { low risk of } \\
\text { bias }\end{array}$ \\
\hline $\begin{array}{l}\text { Memory } \\
\text { T-cells in } \\
\text { tumor tissue }\end{array}$ & 1 & 57 & $\mathrm{~N} / \mathrm{A}$ & & $\begin{array}{c}\text { Memory T-cells } \\
\text { were associated } \\
\text { with PFS in } 1 / 1 \\
\text { studies. }\end{array}$ & & $\begin{array}{c}0 / 1 \text { high risk } 1 / 1 \\
\text { moderate, } 0 / 1 \\
\text { low risk of bias }\end{array}$ \\
\hline $\begin{array}{l}\text { T-cell } \\
\text { inflamed } \\
\text { GEP }\end{array}$ & 1 & 57 & $\mathrm{~N} / \mathrm{A}$ & $\begin{array}{c}\text { GEP was associated } \\
\text { with response in } 1 / 1 \\
\text { studies. }\end{array}$ & & & $\begin{array}{c}0 / 1 \text { high risk } 1 / 1 \\
\text { moderate, } 0 / 1 \\
\text { low risk of bias }\end{array}$ \\
\hline ctDNA & 1 & 35 & $\mathrm{~N} / \mathrm{A}$ & $\begin{array}{l}\text { ctDNA was } \\
\text { associated with } \\
\text { response in } 1 / 1 \\
\text { studies. }\end{array}$ & $\begin{array}{l}\text { ctDNA was } \\
\text { associated with } \\
\text { PFS in } 1 / 1 \\
\text { studies. }\end{array}$ & $\begin{array}{l}\text { ctDNA was } \\
\text { associated with OS } \\
\text { in } 1 / 1 \text { studies. }\end{array}$ & $\begin{array}{l}\text { 1/1 high risk, } \\
0 / 1 \text { moderate } \\
0 / 1 \text { low risk }\end{array}$ \\
\hline TMB & 1 & 35 & $\mathrm{~N} / \mathrm{A}$ & $\begin{array}{c}\text { TMB was associated } \\
\text { with response in } 1 / 1 \\
\text { studies. }\end{array}$ & & $\begin{array}{l}\text { TMB was not } \\
\text { associated with OS } \\
\text { in } 1 / 1 \text { studies. }\end{array}$ & $\begin{array}{c}1 / 1 \text { high risk, } \\
0 / 1 \text { moderate, } \\
0 / 1 \text { low risk of } \\
\text { bias }\end{array}$ \\
\hline
\end{tabular}

Abbreviations: ctDNA: circulating tumor DNA, GEP: gene expression profiling, IQR: interquartile range, LDH: lactate dehydrogenase, NLR: neutrophil-to-lymphocyte ratio, TCR: T cell receptor, TMB: tumor mutation burden.

Table 4. Summary of most important biomarkers that were studied for mixed cohorts and assessed risk of bias per article.

\begin{tabular}{|c|c|c|c|c|c|c|c|}
\hline Biomarker & $\begin{array}{c}\mathbf{N} \text { of } \\
\text { Studies }\end{array}$ & $\begin{array}{l}\mathrm{N} \text { of } \mathrm{Pa}- \\
\text { tients }\end{array}$ & $\begin{array}{c}\text { Median } \\
\text { Patients per } \\
\text { Study (IQR) }\end{array}$ & Response & PFS & OS & $\begin{array}{c}\text { Quality } \\
\text { Assessment }\end{array}$ \\
\hline TMB & 5 & 861 & $91(68-317)$ & $\begin{array}{l}\text { TMB was } \\
\text { associated with } \\
\text { response in } 4 / 5 \\
\text { studies, not } \\
\text { associated in } 1 / 5 \\
\text { studies. }\end{array}$ & $\begin{array}{l}\text { TMB was } \\
\text { associated with } \\
\text { PFS in } 1 / 2 \\
\text { studies, not } \\
\text { associated in } \\
1 / 2 \text { studies. }\end{array}$ & $\begin{array}{c}\text { TMB was } \\
\text { associated with } \\
\text { OS in } 1 / 4 \\
\text { studies, not } \\
\text { associated in } 3 / 4 \\
\text { studies. }\end{array}$ & $\begin{array}{l}2 / 5 \text { high risk, } \\
3 / 5 \text { moderate, } \\
0 / 5 \text { low risk of } \\
\text { bias }\end{array}$ \\
\hline $\begin{array}{l}\text { PD-L1 } \\
\text { expression } \\
\text { on tumor } \\
\text { cells }\end{array}$ & 5 & 298 & $51(1-84)$ & $\begin{array}{l}\text { PD-L1 expression } \\
\text { was associated } \\
\text { with response in } \\
2 / 3 \text { studies, not } \\
\text { associated in } 1 / 3 \\
\text { studies. }\end{array}$ & $\begin{array}{c}\text { PD-L1 } \\
\text { expression was } \\
\text { not associated } \\
\text { with PFS in } 1 / 1 \\
\text { studies. }\end{array}$ & $\begin{array}{l}\text { PD-L1 was } \\
\text { associated with } \\
\text { OS in } 1 / 4 \\
\text { studies, not } \\
\text { associated with } \\
\text { OS in } 3 / 4 \\
\text { studies. }\end{array}$ & $\begin{array}{c}2 / 5 \text { high risk, } \\
3 / 5 \text { moderate, } \\
0 / 5 \text { low risk of } \\
\text { bias }\end{array}$ \\
\hline $\begin{array}{l}\text { Circulating } \\
\text { tumor cells }\end{array}$ & 3 & 190 & $82(22-86)$ & $\begin{array}{c}\text { CTCs were } \\
\text { associated with } \\
\text { response in } 3 / 3 \\
\text { studies. }\end{array}$ & $\begin{array}{l}\text { CTCs were } \\
\text { associated with } \\
\text { PFS in } 3 / 3 \\
\text { studies. }\end{array}$ & $\begin{array}{l}\text { CTCs were } \\
\text { associated with } \\
\text { OS in } 2 / 2 \\
\text { studies. } \\
\text { LDH was }\end{array}$ & $\begin{array}{c}1 / 3 \text { high risk, } \\
2 / 3 \text { moderate } \\
0 / 3 \text { low risk }\end{array}$ \\
\hline $\mathrm{LDH}$ & 2 & 141 & $71(\mathrm{~N} / \mathrm{A})$ & $\begin{array}{l}\text { LDH was not } \\
\text { associated with } \\
\text { response in } 1 / 1 \\
\text { studies. }\end{array}$ & $\begin{array}{c}\text { LDH was } \\
\text { associated with } \\
\text { PFS in } 1 / 2 \\
\text { studies. }\end{array}$ & $\begin{array}{l}\text { associated with } \\
\text { OS in } 1-2 \text { studies, } \\
\text { not associated } \\
\text { with OS in } 1 / 2 \\
\text { studies. }\end{array}$ & $\begin{array}{c}0 / 2 \text { high risk, } \\
2 / 2 \text { moderate, } \\
0 / 2 \text { low risk of } \\
\text { bias }\end{array}$ \\
\hline
\end{tabular}


Table 4. Cont.

\begin{tabular}{|c|c|c|c|c|c|c|c|}
\hline Biomarker & $\begin{array}{c}\mathbf{N} \text { of } \\
\text { Studies }\end{array}$ & $\begin{array}{l}\mathrm{N} \text { of } \mathrm{Pa}- \\
\text { tients }\end{array}$ & $\begin{array}{c}\text { Median } \\
\text { Patients per } \\
\text { Study (IQR) }\end{array}$ & Response & PFS & OS & $\begin{array}{c}\text { Quality } \\
\text { Assessment }\end{array}$ \\
\hline $\begin{array}{l}\text { Neoantigen } \\
\text { load (NAL) }\end{array}$ & 2 & 423 & 212 (N/A) & $\begin{array}{l}\text { NAL was } \\
\text { associated with } \\
\text { response in } 1 / 2 \\
\text { studies, not } \\
\text { associated in } 1 / 2 \\
\text { studies. }\end{array}$ & & $\begin{array}{c}\text { NAL was } \\
\text { associated with } \\
\text { OS in } 1 / 2 \\
\text { studies, not } \\
\text { associated with } \\
\text { OS in } 1 / 2 \\
\text { studies. }\end{array}$ & $\begin{array}{c}2 / 2 \text { high risk, } \\
0 / 2 \text { moderate, } \\
0 / 2 \text { low risk of } \\
\text { bias }\end{array}$ \\
\hline $\begin{array}{l}\text { TILs in } \\
\text { tumor } \\
\text { tissue }\end{array}$ & 2 & 123 & $62(\mathrm{~N} / \mathrm{A})$ & $\begin{array}{l}\text { TILs were } \\
\text { associated with } \\
\text { response in } 1 / 1 \\
\text { studies. }\end{array}$ & $\begin{array}{l}\text { TILs were not } \\
\text { associated with } \\
\text { PFS in } 1 / 1 \\
\text { studies. }\end{array}$ & $\begin{array}{c}\text { TILs were } \\
\text { associated with } \\
\text { OS in } 2 / 2 \\
\text { studies. }\end{array}$ & $\begin{array}{c}1 / 2 \text { high risk } \\
1 / 2 \text { moderate, } \\
0 / 1 \text { low risk of } \\
\text { bias }\end{array}$ \\
\hline $\begin{array}{l}\text { Gut micro- } \\
\text { biomes }\end{array}$ & 2 & 66 & $33(\mathrm{~N} / \mathrm{A})$ & $\begin{array}{l}\text { Gut microbiomes } \\
\text { were associated } \\
\text { with response in } \\
2 / 2 \text { studies. }\end{array}$ & & & $\begin{array}{c}0 / 2 \text { high risk, } \\
2 / 2 \text { moderate, } \\
0 / 2 \text { low risk }\end{array}$ \\
\hline NLR & 1 & 32 & $\mathrm{~N} / \mathrm{A}$ & $\begin{array}{l}\text { NLR was not } \\
\text { associated with } \\
\text { response in } 1 / 1 \\
\text { studies. }\end{array}$ & & & $\begin{array}{c}1 / 1 \text { high risk, } \\
0 / 1 \text { moderate } \\
0 / 1 \text { low risk of } \\
\text { bias }\end{array}$ \\
\hline $\begin{array}{l}\text { Tregs in } \\
\text { tumor } \\
\text { tissue }\end{array}$ & 1 & 32 & $\mathrm{~N} / \mathrm{A}$ & & & $\begin{array}{l}\text { Tregs were not } \\
\text { associated with } \\
\text { OS in } 1 / 1 \\
\text { studies. }\end{array}$ & $\begin{array}{c}1 / 1 \text { high risk, } \\
0 / 1 \text { moderate } \\
0 / 1 \text { low risk of } \\
\text { bias }\end{array}$ \\
\hline
\end{tabular}

Abbreviations: IQR: interquartile range, LDH: lactate dehydrogenase, NLR: neutrophil-to-lymphocyte ratio, TILs: tumor-infiltrating lymphocytes, TMB: tumor mutation burden, Tregs: regulatory T cells.

\subsection{Peripheral Blood Biomarkers}

In total, we identified 72 unique blood biomarkers in 162 different publications (Figure 2, Tables S3-S6). Soluble blood biomarkers that were frequently investigated were LDH, leukocyte counts including lymphocytes, neutrophils, eosinophils and the ratio between these cytology markers, myeloid-derived suppressor cells (MDSCs and subset monocytic MDSCs (moMDSCs)), natural killer (NK) cells, systemic inflammation markers such as cytokines and S100, and circulating tumor cells (CTCs). LDH was the most extensively studied marker in 5149 patients. In the majority of all studies a correlation with OS or PFS was found, but LDH was only associated with response in 9/23 reports. Four of these papers confirmed the association between LDH and OS or PFS in a separate validation cohort [15-18]. Weide and colleagues [18] investigated LDH in the largest cohort $(N=616)$ and demonstrated that a high LDH at baseline was associated with worse OS in both a discovery and confirmation cohort of melanoma patients treated with pembrolizumab.

Neutrophil-to-lymphocyte ratio (NLR) was investigated in 2605 patients and high NLR correlated with poor PFS in 9/10 studies and poor OS in 14/17 studies, whereas a correlation with response was found in $6 / 10$ studies. The largest prospective cohort that studied NLR was the study of Ferrucci and colleagues [19]. In 720 melanoma patients treated with ipilimumab, they found that an elevated NLR at baseline was associated with both OS and PFS, but response was not investigated in this study. Only two studies validated the association between NLR and OS in a separate cohort [20,21]. 

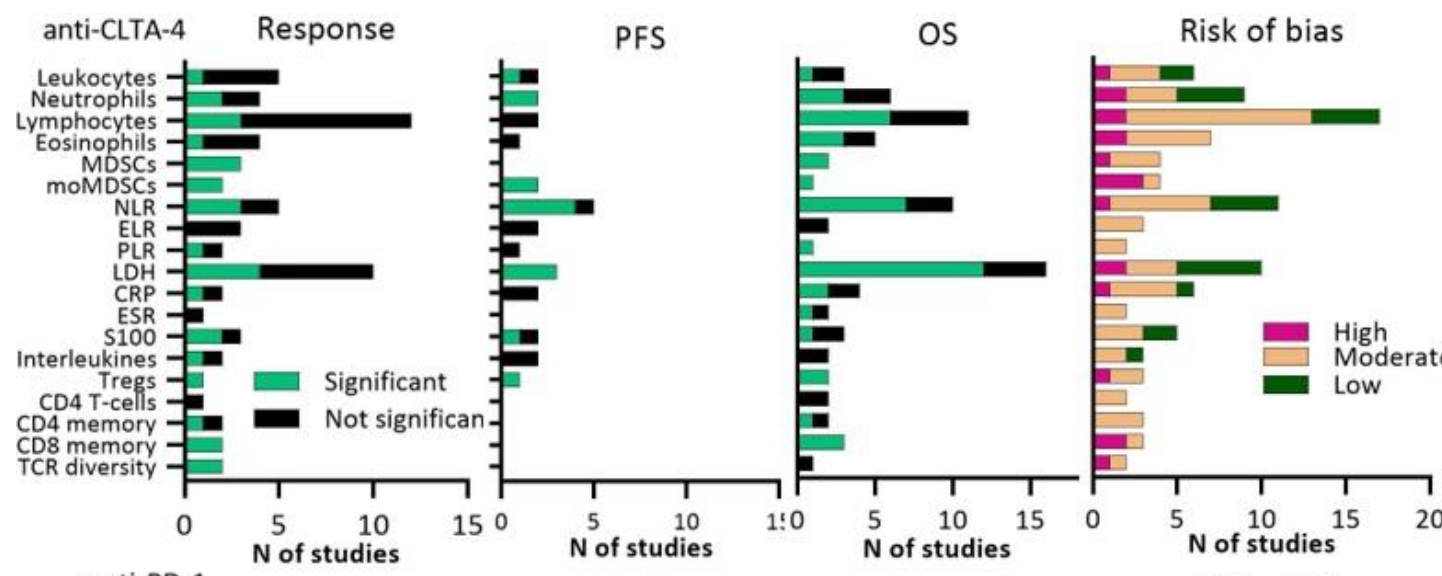

Patients in study
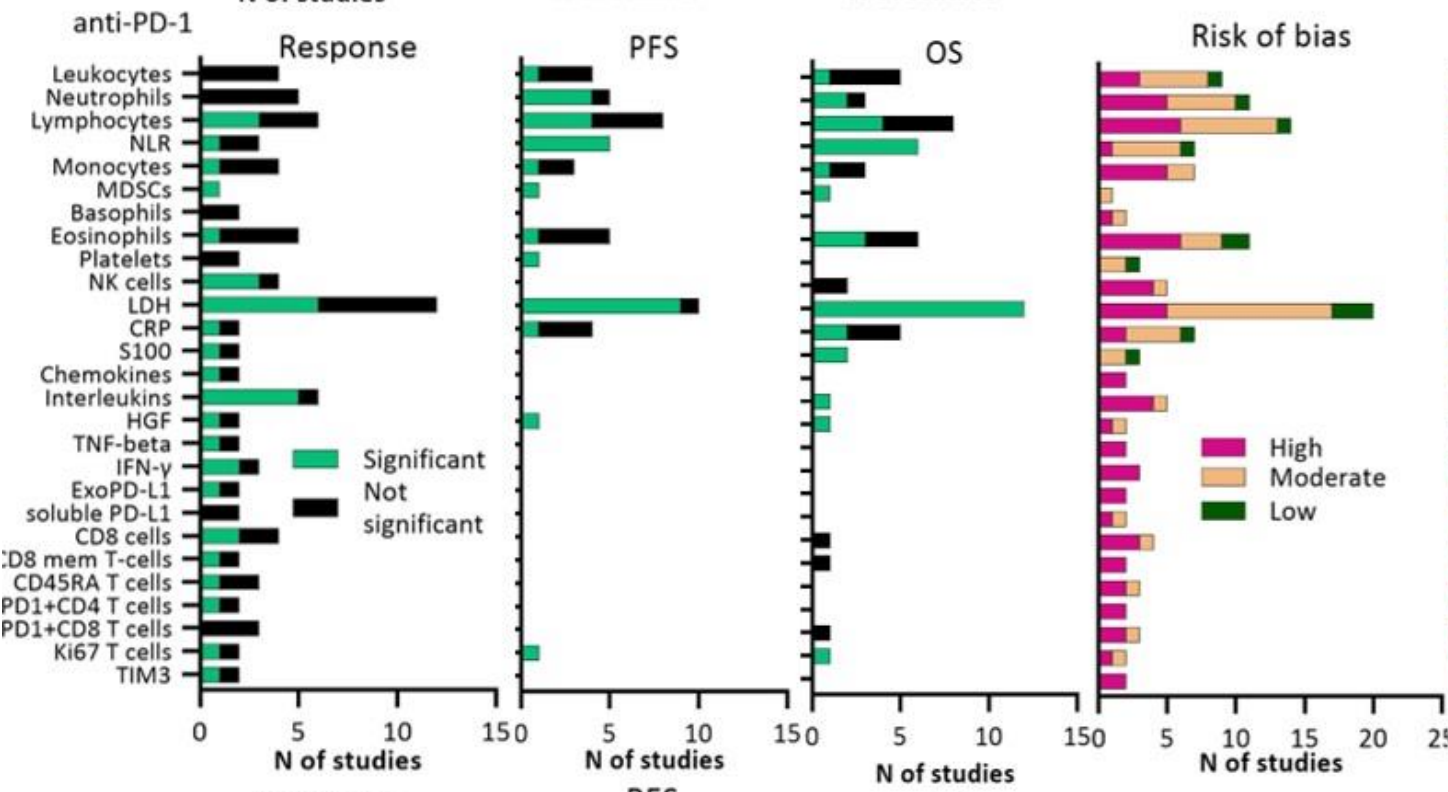

Patients

Mixed cohorts Response

PFS

OS
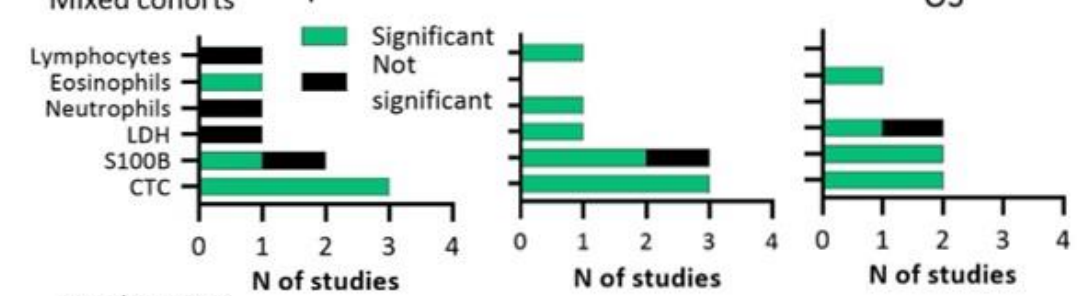

Risk of bias

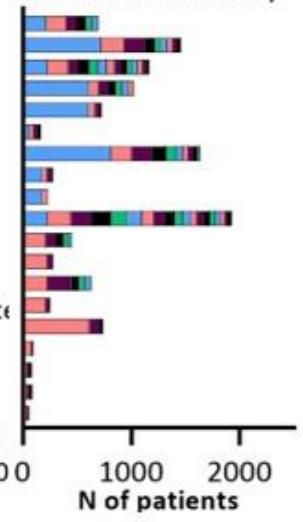

Combination Response
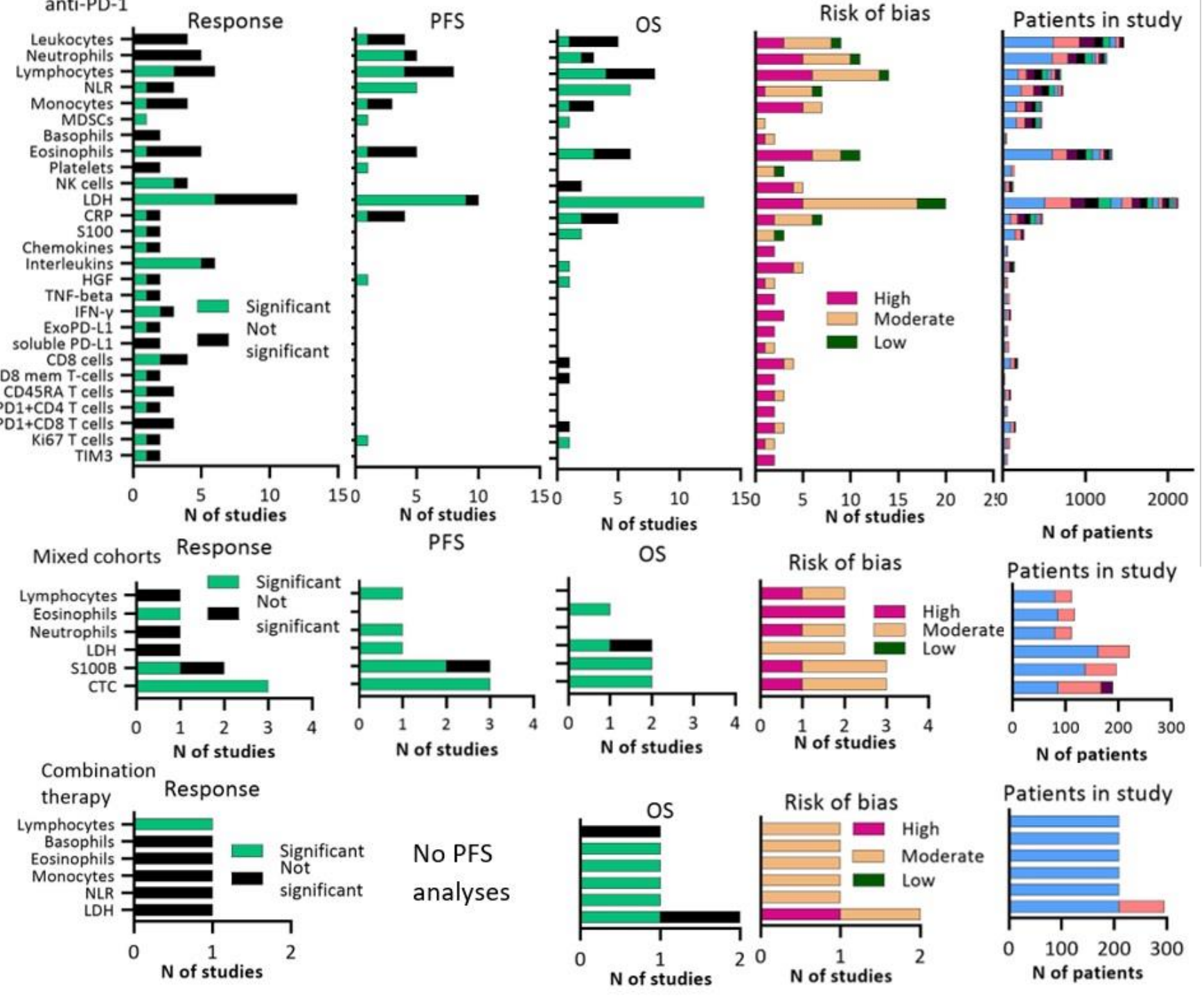

Figure 2. Graphs showing number of studies that were significantly associated or not associated to response, PFS, OS plus risk of bias per article for blood biomarkers. Right part of figure shows total number of included patients. A significant response was defined as $p \leq 0.05$. Abbreviations; CRP:C-reactive Protein, CTC: circulating tumor cells, ELR: eosinophil-tolymphocyte ratio, ESR: erythrocyte sedimentation ratio, HGF: hepatocyte growth factor, LDH: lactate dehydrogenase, NLR: neutrophil-to-lymphocyte ratio, PLR: platelet-to-lymphocyte ratio, Tregs: regulatory T-cells. 
Low MDSC frequencies were an indication for response and OS in all anti-CTLA-4 studies $(N=4)$ and anti-PD-1 studies $(N=1)$, with a moderate risk of bias in $4 / 5$ studies [16,22-24]. MoMDSCs were the only blood parameter that inversely correlated with both response and survival in all anti-CTLA-4 cohorts $(N=4)$, but average risk of bias was high and sample sizes were relatively small (median patients per analysis $=39$, interquartile range (IQR) 32-55) [25-28].

Various pro- and anti-inflammatory cytokines, such as interleukins and chemokines, were analyzed and yielded a few significant correlations for response. Risk of bias was high for the majority of the reports. We also found inconsistent results for S100B and S100A8/9, which are calcium-binding proteins that are increased in melanoma patients. NK cells were associated with response to anti-PD-1 antibodies in 3/4 studies, but not associated with response to anti-CTLA-4 antibodies. Risk of bias was high in $4 / 6$ studies. CTCs or circulating tumor DNA were predominantly studied in mixed therapy cohorts $(N=3)$. Other publications investigated CTCs in anti-PD-1 therapy $(N=1)$, and combination therapy $(N=1)$. Four of these reports were based on prospective cohorts. All reports found an association between a decrease in CTCs and response, OS and PFS and 3/5 analyses were considered a moderate risk of bias [7,29-32].

Biomarkers that play a role in systemic T-cell regulation and activation, such as CD4+, CD45RA+ or CD8+ (effector) memory T cells, regulatory T cells (Tregs), PD-L1+ expression on T cells, T cell repertoire (TCR), and TIM3 or LAG-3 expression on T-cells were investigated in several reports. Increased CD8+ effector memory T cells, associated with long-lived anti-tumor immunity, positively correlated with response in $3 / 4$ studies and with OS in 3/4 studies, but a median of 20 patients per analysis (IQR 13-36,5) were included which resulted in a high risk of bias in 4/5 studies [25,33-36]. A significant correlation between response and TCR diversity in peripheral blood was found in all anti-CLTA-4 $(N=2)$ and anti-PD-1 $(N=1)$ cohorts, but again sample sizes were too small to draw firm conclusions $[37,38]$. PD-1 expression on CD4 or CD8 cells was not associated with response in most (4/5) of the studies (median patients per study $=$ IQR 30-113.5) [33,39-42]. We observed different results for serum Tregs, as they correlated with response in anti-CLTA-4 treated cohorts $(N=1)$ with melanoma patients, but not in anti-PD-1 treated melanoma patients $(N=1)[16,43,44]$.

\subsection{Tumor Biomarkers}

In the tumor tissue-based studies, we identified 55 different biomarkers in 78 publications (Figure 3, Tables S7-S10). Tumor biomarkers that were studied included specific mutations (BRAF, NRAS, cKIT), differential expressed genes included in a gene expression profiling (GEP) score, TMB or neoantigen load (NAL), various T-cell regulation subsets (memory T-cells, regulatory T-cells, TILs), and other immune factors such as perforin or granzyme A or B. Immunohistochemical detected PD-L1 expression on tumor cells was widely studied in a total of 2416 patients. In the anti-PD-1 and mixed therapy cohorts, PD-L1 positivity on pre-treatment tumor cells was correlated with clinical benefit in 9/14 analyses, PFS in 2/5 analyses and OS in 4/8 analyses, although $64 \%$ of the studies were estimated as having a high risk of bias. In a large cohort of 405 melanoma patients who were treated with pembrolizumab, Daud et al. [10] showed that the highest response rates (53-57\%) were found for PD-L1 positive tumors (corresponding to $\geq 10 \%$ staining), whereas patients with PD-L1 negative tumors (corresponding to $<1 \%$ staining) showed response rates of $8-12 \%$. 


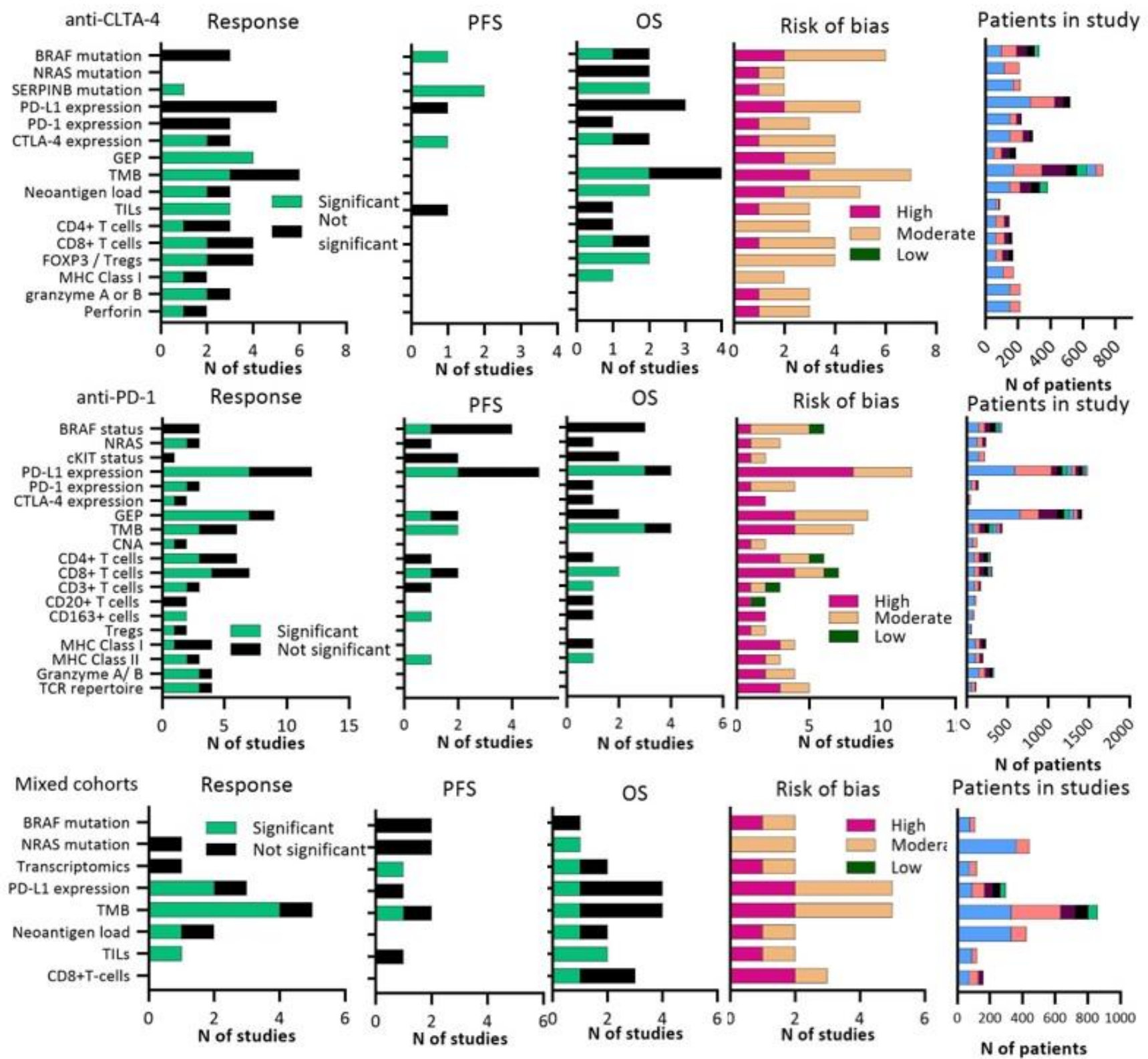

Figure 3. Graphs showing number of studies that were significantly associated or not associated to response, PFS, OS plus risk of bias per article for tumor tissue biomarkers. Right part of figure shows total number of included patients. A significant response was defined as $p \leq 0.05$. Abbreviations; GEP: gene expression profiling, MHC: major histocompatibility complex, TILs: tumor-infiltrating lymphocytes, TCR: T-cell receptor, TMB: tumor mutation burden, Tregs: regulatory T-cells.

TMB and NAL, predominantly resulting from non-synonymous somatic mutations, might also play a role in response to ICIs. Conflicting results were found for TMB, as this biomarker was associated with response in 10/18 studies. More than half (61\%) of these studies were assessed as high risk of bias and only two studies investigated TMB in a validation cohort $[45,46]$. Wood and colleagues investigated TMB in a large cohort $(N=302)$ with both anti-PD-1 and anti-CLTA-4 treated patients, and found no correlation between TMB and response [47]. However, TMB did correlate with response in a cohort of 150 melanoma patients treated with ipilimumab [48]. In anti-CTLA-4 and anti-PD-1 treated patients, responders had a higher NAL at baseline in 3/4 studies, but risk of bias was high in $3 / 4$ studies $[46,48-50]$.

We found 14 studies that calculated a T-cell inflamed GEP score, composed of different inflammatory genes related to T-cell surface markers, antigen presentation, chemokines, 
cytolytic activity, and adaptive immune resistance. T-cell inflamed GEP scores were associated with response in 7/9 of the anti-PD-1 cohorts and 4/4 of the anti-CTLA-4 cohorts, with high $(42 \%)$ and moderate $(58 \%)$ risk of bias reports [36,48,50-61]. Hamid and colleagues [53] investigated an 18-gene T-cell inflamed GEP, which consisted of IFNresponsive genes and was developed in a previous study with 81 melanoma patients treated with pembrolizumab [61] in a large phase $\mathrm{Ib}$ clinical trial $(N=655)$. Their findings revealed an association between GEP score and response to pembrolizumab in both treatment-naïve and treatment-exposed patients. A third study confirmed the association between the 18-gene GEP and response to pembrolizumab in a separate cohort $(N=89)$ [52]. Other studies investigated different immune-related genes in studies with smaller sample sizes.

Intratumor T-cell activation and regulation markers were evaluated in several reports. All studies $(N=6)$ investigating association between TILs and response in anti-CLTA-4, anti-PD-1 and mixed therapy cohorts found significant results and most studies (67\%) were assessed as moderate risk of bias [9,50,62-65]. We found conflicting results for specific T-cell markers, such as CD3+, CD4+ and CD8+ T-cells.

\subsection{Gut Microbiome}

We found three prospective cohorts that investigated potential biomarkers in human stool of 170 melanoma patients in total. All three studies found an association between response and the gut microbiome and had a high or moderate risk of bias. Among responders, microbiomes were enriched with species such as Faecalibacterium and Bacteroidales [66-68]. Oral microbiomes were not associated with response (Table S11).

\section{Discussion}

In this systematic review, we summarize predictive blood, tumor and fecal biomarkers for ICIs in melanoma patients (Figure 4). Our data show that an impressive number of studies have searched for potential biomarkers, but the average predictive quality is moderate, heterogeneity between studies is large, and only a few biomarkers were validated in a separate cohort.

LDH was the most extensively studied blood biomarker and reflects cancer metabolic activity. LDH was mainly associated with OS and PFS but less with response, suggesting that high LDH is a prognostic rather than predictive marker. Similarly, a high NLR predicts a poor prognosis moreso than a low response rate. Immune cells with suppressive functions, such as MDSCs, monocytic MDSCs or Tregs, were more often associated with response in ICI-treated patients. Higher frequencies of Tregs, which express FoxP3, were predominantly associated with response to anti-CTLA-4 therapy, but not to anti-PD-1 antibodies. This is consistent with the mechanism of action of anti-CTLA-4, as preclinical murine studies previously showed that Tregs represent direct target cells to anti-CTLA-4 therapy due to their CTLA-4 expression [69,70]. Furthermore, Martens and colleagues showed that low MDSCs were indicators of benefit for ipilimumab in a development and validation cohort, suggesting that patients with an immune response suppressed by means other than myeloid cells (e.g., Tregs) are more likely to respond to anti-CLTA-4 therapy [16].

TCR diversity in relation to ICIs has also been intensively studied, and several studies have shown that anti-CTLA-4 broadens the peripheral TCR repertoire, whereas anti-PD-1 expands some T-cell clones, which results in a skewed TCR repertoire [71,72]. A high TCR diversity with reduced clonal loss at baseline was reported in responding patients, but few patients were included in these studies. Similarly, CD8+ memory T-cells and CTCs might facilitate adequate patient selection for ICIs, but larger sample sizes are needed to test this hypothesis. 


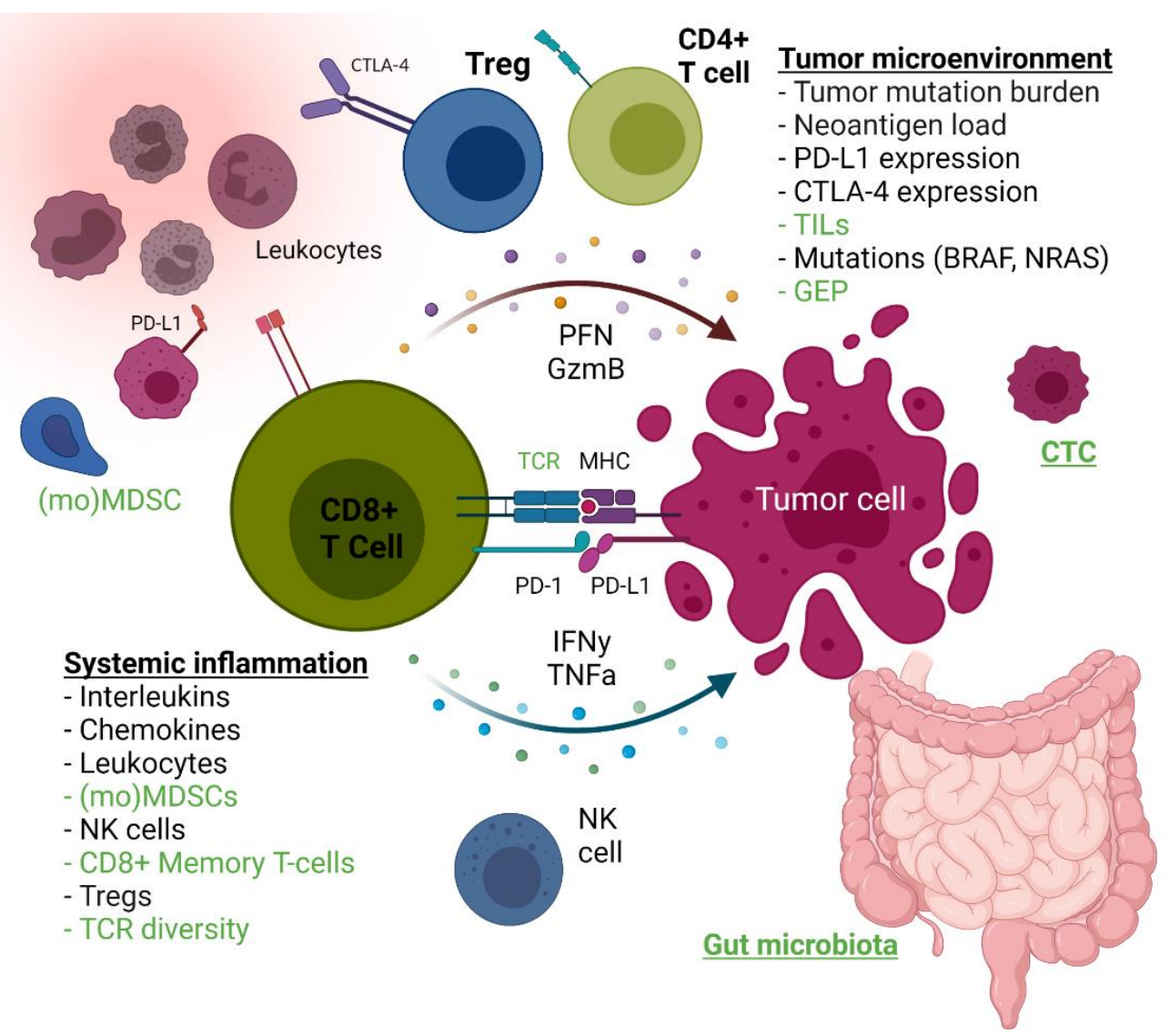

Figure 4. Overview of predictive biomarkers for response to ICIs. Biomarkers that were associated with response in most studies are marked green and biomarkers that were not associated with response in most studies are marked black. T cell infiltration markers, tumor cell microenvironment and gut microbiota. Abbreviations; CTC: circulating tumor cells, GzmB: Granzyme B, MDSCs: myeloidderived suppressor cells, MHC: major histocompatibility complex, NK: natural killer, PFN: perforin, Tregs: regulatory T cells. Created with BioRender (BioRender.com, accessed on 24 November 2021).

In tumor-tissue based publications, PD-L1 expression on pre-treatment tumor samples was the most frequently studied biomarker. In the majority of the anti-PD-1 and mixed therapy cohorts, PD-L1 expression on tumor cells correlated with response. However, PD-L1-negative patients also showed durable responses, suggesting that a negative finding is of limited significance, which justifies why PD-L1 has not been implemented as a biomarker for the treatment of melanoma. Moreover, interpretation of the publications was difficult due to different immunohistochemistry methods and definitions of positive staining. Inconsistent results were also found for the predictive value of TMB. For example, Johnson and colleagues [45] showed that responders had a high mutational load, but TMB only correlated with OS in a report by Hugo et al. [57]. Better studies with harmonized thresholds are needed to further assess both TMB and neoantigen load as predictors in melanoma patients. TILs and TIL subsets were frequently studied, and although TIL numbers were clearly associated with response to ICIs, inconclusive results were described for the TIL subsets. Therefore, further research of TILs is required as well.

An emerging concept is the analysis of the tumor immune microenvironment in the form of T-cell inflamed GEP. T-cell inflamed GEP in tumor tissue was predictive for response in most studies. Most GEP signatures are characterized by, among other things, the upregulation of IFN- $\gamma$ signaling. As the IFN- $\gamma$ signaling pathway can induce the expression of PD-L1 and PD-L2 on tumor cells and macrophages, this signature is critical for antitumor immunity. A promising thing about GEP is its ability to integrate the complex biology of multiple microenvironmental features in comparison with a single biomarker. 
As mentioned in the results section, an 18-gene T-cell inflamed GEP was associated with response to pembrolizumab in three studies, one of which was a large trial, indicating its suitability for clinical implementation. Indeed, robust and reproducible genomic-based platforms will be needed to implement T-cell inflamed GEP in daily oncology care.

Additionally, biomarkers in the intestinal microbiota are emerging to predict response to ICIs. Three studies showed that responders had an abundance of several bacterial species in the gut microbiome. Gopalakrishnan and colleagues therefore proposed that a "favorable" gut microbiome modulates anti-tumor immune responses due to increased antigen presentation and improved effector T-cell function [66]. Interestingly, several clinical trials are currently investigating the alteration of gut microbiota, e.g., with modified bacteria as adjuvant therapy for ICIs, implying that the microbiome might also be a possible target for cancer treatment.

We conducted an extensive literature search to identify predictive biomarkers, reported both positive and negative study results, and performed an adequate quality assessment. To our knowledge, this is the first formal systematic review since 2017 that updates current literature about both anti-CLTA-4, anti-PD-1 and combination therapy in melanoma patients. Compared to the review by Jessurun and colleagues [73], more studies that analyzed biomarkers for anti-PD-1 or combination therapy $(N=81)$ have emerged. Two other important strengths of our review are the large number of publications that we included and the formal bias assessment that we performed for all studies. Moreover, our tables summarizing outcomes per biomarker are a helpful tool for both clinical oncologists and researchers to check if a specific biomarker has been studied before.

Of course, this review has its limitations. First, different thresholds, study methods and adjustment for confounders resulted in high heterogeneity. Second, our review comprises a few Japanese studies that included not only cutaneous melanoma patients but acral and uveal melanoma as well, thereby studying a heterogenous population. Outcomes in Japanese patients might thus be influenced due to different tumor types. Third, as mentioned before, validation of identified predictive markers was often lacking. Last, study results from four publications were too complex and too extensive to accurately summarize all outcomes in our tables [74-77].

Despite previous studies addressing the role of race, lifestyle habits and metabolic disorders as important confounders on the outcome of ICIs [78], none of the included publications adjusted results for these confounders. Future studies on predictive biomarkers should therefore take into account these confounders in their analyses. Regarding gender, male patients were slightly overrepresented in most studies. This overrepresentation might result from a higher incidence of melanoma in males.

While a substantial amount of developmental research on biomarkers for immunotherapy has been published in the past decade, validation studies are still limited. Instead of continuing the search for new biomarkers, future research should focus instead on validating existing biomarkers in large sample sizes.

\section{Conclusions}

Biomarkers for ICIs in melanoma patients are widely studied, and several biomarkers, such as (monocytic) MDSCs, TCR diversity, CTCs, TILs, T-cell inflamed GEP, and gut microbiomes, are associated with response to ICIs. Of these, only T-cell inflamed GEP was predictive for response in a large clinical trial and validated in two studies, representing a promising biomarker for clinical practice. Most studies carried a high or moderate risk of bias due to small sample sizes, no adjustment for confounders and no validation in a separate cohort. Therefore, large prospective studies with comparable thresholds that are adjusted for relevant confounders and validation are warranted to confirm their predictive utility and thereby further personalize immunotherapy treatment. 
Supplementary Materials: The following are available online at https: / www.mdpi.com/article/ 10.3390/cancers13246366/s1, Table S1: Study characteristics of all included reports and quality assessment, Table S2: Outcomes of all included reports, Table S3: Peripheral blood biomarkers that were studied for anti-CLTA-4 therapy, Table S4: Peripheral blood biomarkers that were studied for anti-PD-1 therapy, Table S5: Peripheral blood biomarkers that were studied for mixed cohorts, Table S6: Peripheral blood biomarkers that were studied for combination therapy, Table S7: Tumor biomarkers that were studied for anti-CLTA-4 therapy, Table S8: Tumor biomarkers that were studied for anti-PD-1 therapy, Table S9: Tumor biomarkers that were studied for mixed cohorts, Table S10: Tumor biomarkers that were studied for combination therapy, Table S11: Fecal biomarkers that were studied for all ICIs.

Author Contributions: Conceptualization N.A.d.G.; methodology, N.A.d.G., J.E.A.P.; investigation, J.C.B., N.A.d.G., J.E.A.P.; data curation, J.C.B., N.A.d.G., J.E.A.P.; writing-original draft preparation, J.C.B.; writing-review and editing, all authors; visualization, all authors; supervision, N.A.d.G., J.E.A.P.; funding acquisition, N.A.d.G. All authors have read and agreed to the published version of the manuscript.

Funding: This work was supported by a personal research grant of the last author, provided by the Dutch Research Council (NWO-ZonMW).

Conflicts of Interest: The funders had no role in the design of the study; in the collection, analyses, or interpretation of data; in the writing of the manuscript, or in the decision to publish the results.

\section{References}

1. Integraal Kankercentrum Nederland. Cijfers over Kanker. Available online: www.cijfersoverkanker.nl (accessed on 23 July 2021).

2. Pisibon, C.; Ouertani, A.; Bertolotto, C.; Ballotti, R.; Cheli, Y. Immune Checkpoints in Cancers: From Signaling to the Clinic. Cancers 2021, 13, 4573. [CrossRef] [PubMed]

3. Hodi, F.S.; O’Day, S.J.; McDermott, D.F.; Weber, R.W.; Sosman, J.A.; Haanen, J.B.; Gonzalez, R.; Robert, C.; Schadendorf, D.; Hassel, J.C.; et al. Improved survival with ipilimumab in patients with metastatic melanoma. N. Engl. J. Med. 2010, 363, 711-723. [CrossRef] [PubMed]

4. Robert, C.; Schachter, J.; Long, G.V.; Arance, A.; Grob, J.J.; Mortier, L.; Daud, A.; Carlino, M.S.; McNeil, C.; Lotem, M.; et al. Pembrolizumab versus Ipilimumab in Advanced Melanoma. N. Engl. J. Med. 2015, 372, 2521-2532. [CrossRef]

5. Larkin, J.; Chiarion-Sileni, V.; Gonzalez, R.; Grob, J.J.; Cowey, C.L.; Lao, C.D.; Schadendorf, D.; Dummer, R.; Smylie, M.; Rutkowski, P.; et al. Combined Nivolumab and Ipilimumab or Monotherapy in Untreated Melanoma. N. Engl. J. Med. 2015, 373, 23-34. [CrossRef] [PubMed]

6. Weber, J.; Mandala, M.; Del Vecchio, M.; Gogas, H.J.; Arance, A.M.; Cowey, C.L.; Dalle, S.; Schenker, M.; Chiarion-Sileni, V.; Marquez-Rodas, I.; et al. Adjuvant Nivolumab versus Ipilimumab in Resected Stage III or IV Melanoma. N. Engl. J. Med. 2017, 377, 1824-1835. [CrossRef] [PubMed]

7. Forschner, A.; Battke, F.; Hadaschik, D.; Schulze, M.; Weißgraeber, S.; Han, C.T.; Kopp, M.; Frick, M.; Klumpp, B.; Tietze, N.; et al. Tumor mutation burden and circulating tumor DNA in combined CTLA-4 and PD-1 antibody therapy in metastatic melanomaResults of a prospective biomarker study. J. Immunother. Cancer 2019, 7, 180. [CrossRef] [PubMed]

8. Diem, S.; Kasenda, B.; Spain, L.; Martin-Liberal, J.; Marconcini, R.; Gore, M.; Larkin, J. Serum lactate dehydrogenase as an early marker for outcome in patients treated with anti-PD-1 therapy in metastatic melanoma. Br. J. Cancer 2016, 114, 256-261. [CrossRef] [PubMed]

9. Diem, S.; Hasan, A.O.; Ackermann, C.J.; Bomze, D.; Koelzer, V.H.; Jochum, W.; Speiser, D.E.; Mertz, K.D.; Flatz, L. Tumor infiltrating lymphocytes in lymph node metastases of stage III melanoma correspond to response and survival in nine patients treated with ipilimumab at the time of stage IV disease. Cancer Immunol. Immunother. 2018, 67, 39-45. [CrossRef] [PubMed]

10. Daud, A.I.; Wolchok, J.D.; Robert, C.; Hwu, W.-J.; Weber, J.S.; Ribas, A.; Hodi, F.S.; Joshua, A.; Kefford, R.; Hersey, P.; et al. Programmed Death-Ligand 1 Expression and Response to the Anti-Programmed Death 1 Antibody Pembrolizumab in Melanoma. J. Clin. Oncol. 2016, 34, 4102-4109. [CrossRef] [PubMed]

11. Ribas, A.; Kefford, R.; Marshall, M.A.; Punt, C.J.; Haanen, J.B.; Marmol, M.; Garbe, C.; Gogas, H.; Schachter, J.; Linette, G.; et al. Phase III randomized clinical trial comparing tremelimumab with standard-of-care chemotherapy in patients with advanced melanoma. J. Clin. Oncol. 2013, 31, 616-622. [CrossRef] [PubMed]

12. Moher, D.; Liberati, A.; Tetzlaff, J.; Altman, D.G.; PRISMA Group. Preferred reporting items for systematic reviews and meta-analyses: The PRISMA statement. BMJ 2009, 339, b2535. [CrossRef]

13. Riley, R.D.; Moons, K.G.M.; Snell, K.I.E.; Ensor, J.; Hooft, L.; Altman, D.G.; Hayden, J.; Collins, G.S.; Debray, T.P.A. A guide to systematic review and meta-analysis of prognostic factor studies. BMJ 2019, 364, k4597. [CrossRef] [PubMed]

14. Page, M.J.; Moher, D.; Bossuyt, P.M.; Boutron, I.; Hoffmann, T.C.; Mulrow, C.D.; Shamseer, L.; Tetzlaff, J.M.; Akl, E.A.; Brennan, S.E.; et al. PRISMA 2020 explanation and elaboration: Updated guidance and exemplars for reporting systematic reviews. BMJ 2021, 372, n160. [CrossRef] 
15. Kelderman, S.; Heemskerk, B.; Van Tinteren, H.; Brom, R.R.H.V.D.; Hospers, G.; Eertwegh, A.J.M.V.D.; Kapiteijn, E.; De Groot, J.W.B.; Soetekouw, P.; Jansen, R.L.; et al. Lactate dehydrogenase as a selection criterion for ipilimumab treatment in metastatic melanoma. Cancer Immunol. Immunother. 2014, 63, 449-458. [CrossRef] [PubMed]

16. Martens, A.; Wistuba-Hamprecht, K.; Geukes Foppen, M.; Yuan, J.; Postow, M.A.; Wong, P.; Romano, E.; Khammari, A.; Dreno, B.; Capone, M.; et al. Baseline Peripheral Blood Biomarkers Associated with Clinical Outcome of Advanced Melanoma Patients Treated with Ipilimumab. Clin. Cancer Res. 2016, 22, 2908-2918. [CrossRef] [PubMed]

17. Valpione, S.; Martinoli, C.; Fava, P.; Mocellin, S.; Campana, L.G.; Quaglino, P.; Ferrucci, P.F.; Pigozzo, J.; Astrua, C.; Testori, A.; et al. Personalised medicine: Development and external validation of a prognostic model for metastatic melanoma patients treated with ipilimumab. Eur. J. Cancer 2015, 51, 2086-2094. [CrossRef]

18. Weide, B.; Martens, A.; Hassel, J.C.; Berking, C.; Postow, M.A.; Bisschop, K.; Simeone, E.; Mangana, J.; Schilling, B.; Di Giacomo, A.M.; et al. Baseline Biomarkers for Outcome of Melanoma Patients Treated with Pembrolizumab. Clin. Cancer Res. 2016, 22, 5487-5496. [CrossRef]

19. Ferrucci, P.F.; Ascierto, P.A.; Pigozzo, J.; Del Vecchio, M.; Maio, M.; Antonini Cappellini, G.C.; Guidoboni, M.; Queirolo, P.; Savoia, P.; Mandalà, M.; et al. Baseline neutrophils and derived neutrophil-to-lymphocyte ratio: Prognostic relevance in metastatic melanoma patients receiving ipilimumab. Ann. Oncol. 2016, 27, 732-738. [CrossRef]

20. Ferrucci, P.F.; Gandini, S.; Battaglia, A.; Alfieri, S.; Di Giacomo, A.M.; Giannarelli, D.; Cappellini, G.C.A.; De Galitiis, F.; Marchetti, P.; Amato, G.; et al. Baseline neutrophil-to-lymphocyte ratio is associated with outcome of ipilimumab-treated metastatic melanoma patients. Br. J. Cancer 2015, 112, 1904-1910. [CrossRef]

21. Iivanainen, S.; Ahvonen, J.; Knuuttila, A.; Tiainen, S.; Koivunen, J.P. Elevated CRP levels indicate poor progression-free and overall survival on cancer patients treated with PD-1 inhibitors. ESMO Open 2019, 4, e000531. [CrossRef]

22. Bjoern, J.; Juul Nitschke, N.; Zeeberg Iversen, T.; Schmidt, H.; Fode, K.; Svane, I.M. Immunological correlates of treatment and response in stage IV malignant melanoma patients treated with Ipilimumab. OncoImmunology 2016, 5, e1100788. [CrossRef]

23. Meyer, C.; Cagnon, L.; Costa-Nunes, C.M.; Baumgaertner, P.; Montandon, N.; Leyvraz, L.; Michielin, O.; Romano, E.; Speiser, D.E. Frequencies of circulating MDSC correlate with clinical outcome of melanoma patients treated with ipilimumab. Cancer Immunol. Immunother. 2013, 63, 247-257. [CrossRef] [PubMed]

24. Sade-Feldman, M.; Kanterman, J.; Klieger, Y.; Ish-Shalom, E.; Olga, M.; Saragovi, A.; Shtainberg, H.; Lotem, M.; Baniyash, M. Clinical Significance of Circulating CD33+CD11b+HLA-DR - Myeloid Cells in Patients with Stage IV Melanoma Treated with Ipilimumab. Clin. Cancer Res. 2016, 22, 5661-5672. [CrossRef] [PubMed]

25. de Coaña, Y.P.; Wolodarski, M.; Poschke, I.; Yoshimoto, Y.; Yang, Y.; Nyström, M.; Edbäck, U.; Brage, S.E.; Lundqvist, A.; Masucci, G.; et al. Ipilimumab treatment decreases monocytic MDSCs and increases CD8 effector memory T cells in long-term survivors with advanced melanoma. Oncotarget 2017, 8, 21539-21553. [CrossRef] [PubMed]

26. Gebhardt, C.; Sevko, A.; Jiang, H.; Lichtenberger, R.; Reith, M.; Tarnanidis, K.; Holland-Letz, T.; Umansky, L.; Beckhove, P.; Sucker, A.; et al. Myeloid Cells and Related Chronic Inflammatory Factors as Novel Predictive Markers in Melanoma Treatment with Ipilimumab. Clin. Cancer Res. 2015, 21, 5453-5459. [CrossRef]

27. Retseck, J.; Nasr, A.; Lin, Y.; Lin, H.; Mendiratta, P.; Butterfield, L.H.; Tarhini, A.A. Long term impact of CTLA4 blockade immunotherapy on regulatory and effector immune responses in patients with melanoma. J. Transl. Med. 2018, 16, 184. [CrossRef]

28. Weber, J.; Gibney, G.; Kudchadkar, R.R.; Yu, B.; Cheng, P.; Martinez, A.J.; Kroeger, J.; Richards, A.; McCormick, L.; Moberg, V.; et al. Phase I/II Study of Metastatic Melanoma Patients Treated with Nivolumab Who Had Progressed after Ipilimumab. Cancer Immunol. Res. 2016, 4, 345-353. [CrossRef]

29. Hong, X.; Sullivan, R.J.; Kalinich, M.; Kwan, T.T.; Giobbie-Hurder, A.; Pan, S.; LiCausi, J.A.; Milner, J.D.; Nieman, L.T.; Wittner, B.S.; et al. Molecular signatures of circulating melanoma cells for monitoring early response to immune checkpoint therapy. Proc. Natl. Acad. Sci. USA 2018, 115, 2467-2472. [CrossRef]

30. Seremet, T.; Jansen, Y.; Planken, S.; Njimi, H.; Delaunoy, M.; El Housni, H.; Awada, G.; Schwarze, J.K.; Keyaerts, M.; Everaert, H.; et al. Undetectable circulating tumor DNA (ctDNA) levels correlate with favorable outcome in metastatic melanoma patients treated with anti-PD1 therapy. J. Transl. Med. 2019, 17, 1-13. [CrossRef]

31. Keller, L.; Guibert, N.; Casanova, A.; Brayer, S.; Farella, M.; Delaunay, M.; Gilhodes, J.; Martin, E.; Balagué, G.; Favre, G.; et al. Early Circulating Tumour DNA Variations Predict Tumour Response in Melanoma Patients Treated with Immunotherapy. Acta Derm. Venereol. 2019, 99, 206-210. [CrossRef]

32. Lee, J.H.; Long, G.V.; Boyd, S.; Lo, S.; Menzies, A.M.; Tembe, V.; Guminski, A.; Jakrot, V.; Scolyer, R.A.; Mann, G.J.; et al. Circulating tumour DNA predicts response to anti-PD1 antibodies in metastatic melanoma. Ann. Oncol. 2017, 28, 1130-1136. [CrossRef] [PubMed]

33. Wistuba-Hamprecht, K.; Martens, A.; Heubach, F.; Romano, E.; Foppen, M.G.; Yuan, J.; Postow, M.; Wong, P.; Mallardo, D.; Schilling, B.; et al. Peripheral CD8 effector-memory type 1 T-cells correlate with outcome in ipilimumab-treated stage IV melanoma patients. Eur. J. Cancer 2017, 73, 61-70. [CrossRef] [PubMed]

34. Tietze, J.K.; Wilkins, D.E.C.; Sckisel, G.D.; Bouchlaka, M.N.; Alderson, K.L.; Weiss, J.M.; Ames, E.; Bruhn, K.W.; Craft, N.; Wiltrout, R.H.; et al. Delineation of antigen-specific and antigen-nonspecific CD8+ memory T-cell responses after cytokine-based cancer immunotherapy. Blood 2012, 119, 3073-3083. [CrossRef] [PubMed] 
35. Subrahmanyam, P.B.; Dong, Z.; Gusenleitner, D.; Giobbie-Hurder, A.; Severgnini, M.; Zhou, J.; Manos, M.; Eastman, L.M.; Maecker, H.T.; Hodi, F.S. Distinct predictive biomarker candidates for response to anti-CTLA-4 and anti-PD-1 immunotherapy in melanoma patients. J. Immunother. Cancer 2018, 6, 18. [CrossRef] [PubMed]

36. Gide, T.N.; Quek, C.; Menzies, A.M.; Tasker, A.T.; Shang, P.; Holst, J.; Madore, J.; Lim, S.Y.; Velickovic, R.; Wongchenko, M.; et al. Distinct Immune Cell Populations Define Response to Anti-PD-1 Monotherapy and Anti-PD-1/Anti-CTLA-4 Combined Therapy. Cancer Cell 2019, 35, 238-255.e6. [CrossRef] [PubMed]

37. Postow, M.A.; Manuel, M.; Wong, P.; Yuan, J.; Dong, Z.; Liu, C.; Perez, S.; Tanneau, I.; Noel, M.; Courtier, A.; et al. Peripheral T cell receptor diversity is associated with clinical outcomes following ipilimumab treatment in metastatic melanoma. J. Immunother. Cancer 2015, 3, 23. [CrossRef]

38. Hogan, S.A.; Courtier, A.; Cheng, P.F.; Jaberg-Bentele, N.F.; Goldinger, S.M.; Manuel, M.; Perez, S.; Plantier, N.; Mouret, J.-F.; Nguyen-Kim, T.D.L.; et al. Peripheral Blood TCR Repertoire Profiling May Facilitate Patient Stratification for Immunotherapy against Melanoma. Cancer Immunol. Res. 2018, 7, 77-85. [CrossRef] [PubMed]

39. Capone, M.; Fratangelo, F.; Giannarelli, D.; Sorrentino, C.; Turiello, R.; Zanotta, S.; Galati, D.; Madonna, G.; Tuffanelli, M.; Scarpato, L.; et al. Frequency of circulating CD8+CD73+T cells is associated with survival in nivolumab-treated melanoma patients. J. Transl. Med. 2020, 18,1-10. [CrossRef]

40. Graves, M.; CelliMarchett, G.; Van Zyl, B.; Tang, D.; Vilain, R.E.; van der Westhuizen, A.; Bowden, N.A. Monitoring Patient Response to Pembrolizumab With Peripheral Blood Exhaustion Marker Profiles. Front. Med. 2019, 6, 113. [CrossRef]

41. Jacquelot, N.; Roberti, M.P.; Enot, D.P.; Rusakiewicz, S.; Ternès, N.; Jegou, S.; Woods, D.M.; Sodré, A.L.; Hansen, M.; Meirow, Y.; et al. Predictors of responses to immune checkpoint blockade in advanced melanoma. Nat. Commun. 2017, 8 , 592. [CrossRef]

42. Bochem, J.; Zelba, H.; Amaral, T.; Spreuer, J.; Soffel, D.; Eigentler, T.; Wagner, N.B.; Uslu, U.; Terheyden, P.; Meier, F.; et al. Peripheral PD-1+CD56+ T-cell frequencies correlate with outcome in stage IV melanoma under PD-1 blockade. PLoS ONE 2019, 14, e0221301. [CrossRef] [PubMed]

43. Nonomura, Y.; Otsuka, A.; Nakashima, C.; Seidel, J.; Kitoh, A.; Dainichi, T.; Nakajima, S.; Sawada, Y.; Matsushita, S.; Aoki, M.; et al. Peripheral blood Th9 cells are a possible pharmacodynamic biomarker of nivolumab treatment efficacy in metastatic melanoma patients. OncoImmunology 2016, 5, e1248327. [CrossRef]

44. Simeone, E.; Gentilcore, G.; Giannarelli, D.; Grimaldi, A.M.; Caracò, C.; Curvietto, M.; Esposito, A.; Paone, M.; Palla, M.; Cavalcanti, E.; et al. Immunological and biological changes during ipilimumab treatment and their potential correlation with clinical response and survival in patients with advanced melanoma. Cancer Immunol. Immunother. 2014, 63, 675-683. [CrossRef]

45. Johnson, D.B.; Frampton, G.; Rioth, M.J.; Yusko, E.; Xu, Y.; Guo, X.; Ennis, R.C.; Fabrizio, D.; Chalmers, Z.R.; Greenbowe, J.; et al. Targeted Next Generation Sequencing Identifies Markers of Response to PD-1 Blockade. Cancer Immunol. Res. 2016, 4, 959-967. [CrossRef] [PubMed]

46. Snyder, A.; Makarov, V.; Merghoub, T.; Yuan, J.; Zaretsky, J.M.; Desrichard, A.; Walsh, L.A.; Postow, M.A.; Wong, P.; Ho, T.S.; et al. Genetic Basis for Clinical Response to CTLA-4 Blockade in Melanoma. N. Engl. J. Med. 2014, 371, 2189-2199. [CrossRef] [PubMed]

47. Wood, M.A.; Weeder, B.; David, J.; Nellore, A.; Thompson, R.F. Burden of tumor mutations, neoepitopes, and other variants are weak predictors of cancer immunotherapy response and overall survival. Genome Med. 2020, 12, 33. [CrossRef] [PubMed]

48. Vargas, F.A.; Furness, A.J.S.; Litchfield, K.; Joshi, K.; Rosenthal, R.; Ghorani, E.; Solomon, I.; Lesko, M.H.; Ruef, N.; Roddie, C.; et al. Fc Effector Function Contributes to the Activity of Human Anti-CTLA-4 Antibodies. Cancer Cell 2018, 33, 649-663.e4. [CrossRef] [PubMed]

49. Van Allen, E.M.; Miao, D.; Schilling, B.; Shukla, S.A.; Blank, C.; Zimmer, L.; Sucker, A.; Hillen, U.; Foppen, M.H.G.; Goldinger, S.M.; et al. Genomic correlates of response to CTLA-4 blockade in metastatic melanoma. Science 2015, 350, 207-211. [CrossRef] [PubMed]

50. Riaz, N.; Havel, J.; Makarov, V.; Desrichard, A.; Urba, W.J.; Sims, J.S.; Hodi, F.S.; Martín-Algarra, S.; Mandal, R.; Sharfman, W.H.; et al. Tumor and Microenvironment Evolution during Immunotherapy with Nivolumab. Cell 2017, 171, 934-949.e16. [CrossRef] [PubMed]

51. Chen, P.-L.; Roh, W.; Reuben, A.; Cooper, Z.A.; Spencer, C.N.; Prieto, P.A.; Miller, J.P.; Bassett, R.L.; Gopalakrishnan, V.; Wani, K.; et al. Analysis of Immune Signatures in Longitudinal Tumor Samples Yields Insight into Biomarkers of Response and Mechanisms of Resistance to Immune Checkpoint Blockade. Cancer Discov. 2016, 6, 827-837. [CrossRef] [PubMed]

52. Cristescu, R.; Mogg, R.; Ayers, M.; Albright, A.; Murphy, E.; Yearley, J.; Sher, X.; Liu, X.Q.; Lu, H.; Nebozhyn, M.; et al. Pan-tumor genomic biomarkers for PD-1 checkpoint blockade-based immunotherapy. Science 2018, 362, 197. [CrossRef]

53. Hamid, O.; Robert, C.; Daud, A.; Hodi, F.S.; Hwu, W.J.; Kefford, R.; Wolchok, J.D.; Hersey, P.; Joseph, R.; Weber, J.S.; et al. Five-year survival outcomes for patients with advanced melanoma treated with pembrolizumab in KEYNOTE-001. Ann. Oncol. 2019, 30, 582-588. [CrossRef] [PubMed]

54. Karachaliou, N.; Gonzalez-Cao, M.; Crespo, G.; Drozdowskyj, A.; Aldeguer, E.; Gimenez-Capitan, A.; Teixido, C.; Molina-Vila, M.A.; Viteri, S.; De Los Llanos Gil, M.; et al. Interferon gamma, an important marker of response to immune checkpoint blockade in non-small cell lung cancer and melanoma patients. Ther. Adv. Med. Oncol. 2018, 10, 1758834017749748. [CrossRef] [PubMed] 
55. Roh, W.; Chen, P.-L.; Reuben, A.; Spencer, C.N.; Prieto, P.A.; Miller, J.P.; Gopalakrishnan, V.; Wang, F.; Cooper, Z.A.; Reddy, S.M.; et al. Integrated molecular analysis of tumor biopsies on sequential CTLA-4 and PD-1 blockade reveals markers of response and resistance. Sci. Transl. Med. 2017, 9, eaaah3560. [CrossRef] [PubMed]

56. Ji, R.-R.; Chasalow, S.D.; Wang, L.; Hamid, O.; Schmidt, H.; Cogswell, J.; Alaparthy, S.; Berman, D.; Jure-Kunkel, M.; Siemers, N.O.; et al. An immune-active tumor microenvironment favors clinical response to ipilimumab. Cancer Immunol. Immunother. 2011, 61, 1019-1031. [CrossRef] [PubMed]

57. Hugo, W.; Zaretsky, J.M.; Sun, L.; Song, C.; Moreno, B.H.; Hu-Lieskovan, S.; Berent-Maoz, B.; Pang, J.; Chmielowski, B.; Cherry, G.; et al. Genomic and Transcriptomic Features of Response to Anti-PD-1 Therapy in Metastatic Melanoma. Cell 2016, 165, 35-44. [CrossRef] [PubMed]

58. Rodig, S.J.; Gusenleitner, D.; Jackson, D.G.; Gjini, E.; Giobbie-Hurder, A.; Jin, C.; Chang, H.; Lovitch, S.B.; Horak, C.; Weber, J.S.; et al. MHC proteins confer differential sensitivity to CTLA-4 and PD-1 blockade in untreated metastatic melanoma. Sci. Transl. Med. 2018, 10, eaar3342. [CrossRef]

59. Tarhini, A.A.; Lin, Y.; Lin, H.-M.; Vallabhaneni, P.; Sander, C.; LaFramboise, W.; Hamieh, L. Expression profiles of immune-related genes are associated with neoadjuvant ipilimumab clinical benefit. OncoImmunology 2017, 6, e1231291. [CrossRef]

60. Varn, F.S.; Wang, Y.; Cheng, C. A B cell-derived gene expression signature associates with an immunologically active tumor microenvironment and response to immune checkpoint blockade therapy. OncoImmunology 2018, 8, e1513440. [CrossRef]

61. Ayers, M.; Lunceford, J.; Nebozhyn, M.; Murphy, E.; Loboda, A.; Kaufman, D.R.; Albright, A.; Cheng, J.D.; Kang, S.P.; Shankaran, V.; et al. IFN- $\gamma$-related mRNA profile predicts clinical response to PD-1 blockade. J. Clin. Investig. 2017, 127, 2930-2940. [CrossRef] [PubMed]

62. Ribas, A.; Shin, D.; Zaretsky, J.; Frederiksen, J.; Cornish, A.; Avramis, E.; Seja, E.; Kivork, C.; Siebert, J.; Kaplan-Lefko, P.; et al. PD-1 Blockade Expands Intratumoral Memory T Cells. Cancer Immunol. Res. 2016, 4, 194-203. [CrossRef] [PubMed]

63. Wong, P.F.; Wei, W.; Smithy, J.W.; Acs, B.; Toki, M.; Blenman, K.R.; Zelterman, D.; Kluger, H.M.; Rimm, D.L. Multiplex Quantitative Analysis of Tumor-Infiltrating Lymphocytes and Immunotherapy Outcome in Metastatic Melanoma. Clin. Cancer Res. 2019, 25, 2442-2449. [CrossRef] [PubMed]

64. Hamid, O.; Schmidt, H.; Nissan, A.; Ridolfi, L.; Aamdal, S.; Hansson, J.; Guida, M.; Hyams, D.M.; Gómez, H.; Bastholt, L.; et al. A prospective phase II trial exploring the association between tumor microenvironment biomarkers and clinical activity of ipilimumab in advanced melanoma. J. Transl. Med. 2011, 9, 204. [CrossRef]

65. Mastracci, L.; Fontana, V.; Queirolo, P.; Carosio, R.; Grillo, F.; Morabito, A.; Banelli, B.; Tanda, E.; Boutros, A.; Dozin, B.; et al. Response to ipilimumab therapy in metastatic melanoma patients: Potential relevance of CTLA-4+ tumor infiltrating lymphocytes and their in situ localization. Cancer Immunol. Immunother. 2020, 69, 653-662. [CrossRef]

66. Gopalakrishnan, V.; Spencer, C.N.; Nezi, L.; Reuben, A.; Andrews, M.C.; Karpinets, T.V.; Prieto, P.A.; Vicente, D.; Hoffman, K.; Wei, S.C.; et al. Gut microbiome modulates response to anti-PD-1 immunotherapy in melanoma patients. Science 2018, 359, 97-103. [CrossRef]

67. Matson, V.; Fessler, J.; Bao, R.; Chongsuwat, T.; Zha, Y.; Alegre, M.-L.; Luke, J.J.; Gajewski, T.F. The commensal microbiome is associated with anti-PD-1 efficacy in metastatic melanoma patients. Science 2018, 359, 104-108. [CrossRef]

68. Frankel, A.E.; Coughlin, L.A.; Kim, J.; Froehlich, T.W.; Xie, Y.; Frenkel, E.P.; Koh, A. Metagenomic Shotgun Sequencing and Unbiased Metabolomic Profiling Identify Specific Human Gut Microbiota and Metabolites Associated with Immune Checkpoint Therapy Efficacy in Melanoma Patients. Neoplasia 2017, 19, 848-855. [CrossRef]

69. Simpson, T.R.; Li, F.; Montalvo-Ortiz, W.; Sepulveda, M.A.; Bergerhoff, K.; Arce, F.; Roddie, C.; Henry, J.Y.; Yagita, H.; Wolchok, J.D.; et al. Fc-dependent depletion of tumor-infiltrating regulatory T cells co-defines the efficacy of anti-CTLA-4 therapy against melanoma. J. Exp. Med. 2013, 210, 1695-1710. [CrossRef]

70. Selby, M.J.; Engelhardt, J.J.; Quigley, M.; Henning, K.A.; Chen, T.; Srinivasan, M.; Korman, A.J. Anti-CTLA-4 Antibodies of IgG2a Isotype Enhance Antitumor Activity through Reduction of Intratumoral Regulatory T Cells. Cancer Immunol. Res. 2013, 1, 32-42. [CrossRef]

71. Robert, L.; Tsoi, J.; Wang, X.; Emerson, R.; Homet, B.; Chodon, T.; Mok, S.; Huang, R.R.; Cochran, A.J.; Comin-Anduix, B.; et al. CTLA4 Blockade Broadens the Peripheral T-Cell Receptor Repertoire. Clin. Cancer Res. 2014, 20, 2424-2432. [CrossRef] [PubMed]

72. Inoue, H.; Park, J.-H.; Kiyotani, K.; Zewde, M.G.; Miyashita, A.; Jinnin, M.; Kiniwa, Y.; Okuyama, R.; Tanaka, R.; Fujisawa, Y.; et al. Intratumoral expression levels of PD-L1, GZMA, and HLA-A along with oligoclonal T cell expansion associate with response to nivolumab in metastatic melanoma. OncoImmunology 2016, 5, e1204507. [CrossRef]

73. Jessurun, C.A.C.; Vos, J.A.M.; Limpens, J.; Luiten, R.M. Biomarkers for Response of Melanoma Patients to Immune Checkpoint Inhibitors: A Systematic Review. Front. Oncol. 2017, 7, 233. [CrossRef] [PubMed]

74. Woods, D.M.; Laino, A.S.; Winters, A.F.; Alexandre, J.M.; Freeman, D.; Rao, V.; Adavani, S.S.; Weber, J.S.; Chattopadhyay, P.K. Nivolumab and ipilimumab are associated with distinct immune landscape changes and response-associated immunophenotypes. JCI Insight 2020, 5, e137066. [CrossRef] [PubMed]

75. Huang, A.C.; Postow, M.A.; Orlowski, R.J.; Mick, R.; Bengsch, B.; Manne, S.; Xu, W.; Harmon, S.; Giles, J.R.; Wenz, B.; et al. T-cell invigoration to tumour burden ratio associated with anti-PD-1 response. Nature 2017, 545, 60-65. [CrossRef]

76. Chen, H.; Yang, M.; Wang, Q.; Song, F.; Li, X.; Chen, K. The new identified biomarkers determine sensitivity to immune check-point blockade therapies in melanoma. OncoImmunology 2019, 8, 1608132. [CrossRef] [PubMed] 
77. Krieg, C.; Nowicka, M.; Guglietta, S.; Schindler, S.; Hartmann, F.J.; Weber, L.M.; Dummer, R.; Robinson, M.D.; Levesque, M.P.; Becher, B. High-dimensional single-cell analysis predicts response to anti-PD-1 immunotherapy. Nat. Med. 2018, 24, 144-153. [CrossRef]

78. Deshpande, R.P.; Sharma, S.; Watabe, K. The Confounders of Cancer Immunotherapy: Roles of Lifestyle, Metabolic Disorders and Sociological Factors. Cancers 2020, 12, 2983. [CrossRef] 